\title{
Wavelet tools to study intermittency: application to vortex bursting
}

\author{
JORI RUPPERT-FELSOT ${ }^{1,2}$, MARIE FARGE ${ }^{1} \dagger$ \\ AND PHILIPPE PETITJEANS ${ }^{2}$ \\ ${ }^{1}$ LMD-IPSL-CNRS, Ecole Normale Supérieure, 24 rue Lhomond, 75231 Paris Cedex 05, France \\ ${ }^{2}$ PMMH, ESPCI, 10 rue Vauquelin, 75231 Paris Cedex 05, France
}

(Received 9 July 2008; revised 5 May 2009; accepted 5 May 2009)

This paper proposes statistical tools adapted to study highly unsteady and inhomogeneous flows, such as vortex bursting. For this, we use the wavelet representation in which each coefficient keeps track of both location and scale, in contrast to Fourier representation which requires keeping the phase of all coefficients to preserve the spatial structure of the flow. Based on the continuous wavelet transform, we propose several diagnostics, such as the local spectrum and the local intermittency measure. We also use the orthogonal wavelet transform to split each flow realization into coherent and incoherent contributions, which are then analysed independently and from which we define the coherency measure. We apply these wavelet tools to analyse the bursting of a three-dimensional stretched vortex immersed in a steady laminar channel flow. The time evolution of the velocity field is measured by particle image velocimetry during several successive bursts.

\section{Introduction}

Classical statistical tools used to analyse turbulence (i.e. correlation function, structure functions, energy spectrum) assume stationarity and homogeneity of the flow. In this paper, we propose wavelet-based statistical tools which do not require such hypotheses and therefore allow us to study unsteady and inhomogeneous flows. We present several diagnostics to quantify the intermittency of turbulent flows. These new tools are based upon the wavelet representation whose basis functions are well localized in both physical and spectral space. Since Fourier modes are delocalized in physical space, albeit well localized in spectral space, the spatial information cannot be retrieved from a subset of Fourier coefficients, unless one keeps the phase of all of them. In contrast, a subset of wavelet coefficients is sufficient to represent spatial information, since each wavelet coefficient is indexed in scale and also in space. Therefore, filtering the wavelet coefficients preserves the spatial structure of the flow, which is not the case for Fourier filtering.

There exists two kinds of wavelet transform: the continuous wavelet transform (CWT) and the orthogonal wavelet transform (OWT). The first is better suited to analysis, since it unfolds the flow in both space and scale in a continuous fashion. This yields a redundancy of information, which presents the advantage of good readability 
of the continuous wavelet coefficients. However, this overcomplete representation results in a correlation between neighbouring coefficients, which corresponds to the reproducing kernel of the CWT. The OWT overcomes this problem but at the price of loosing translation invariance. Indeed, the orthogonal wavelet coefficients are only defined on a dyadic grid, their scale being sampled by octaves and their position restricted to discrete intervals whose size varies with scale (Farge 1992).

Wavelets have been used since more than 20 years to analyse turbulent flows (Farge \& Rabreau 1988; Farge 1992). The modulus of the continuous wavelet coefficients gives the distribution in space and scale of the variance, together with the local deviation from the mean spectrum. We can thus identify which scales and which locations in physical space contribute significantly to the nonlinear cascade and which ones remain inactive. These analyses have also shown that the strongest wavelet coefficients correspond to the coherent structures observed in both direct numerical simulations (Farge et al. 1992) and laboratory experiments (Ruppert-Felsot et al. 2005). We propose in this paper several wavelet-based diagnostics, such as the local spectrum, local intermittency measure (LIM) and coherency measure (CM), to analyse highly unsteady and inhomogeneous turbulent flows. In particular, we would like to quantify the flow intermittency, to understand how the scaling of the energy spectrum develops and which structures are responsible for it.

In Farge (1992) and Farge et al. (1992) we have shown that the coherent structures compress well into wavelet or wavelet packet bases, namely they could be represented by only few strong coefficients. In contrast, the remaining weaker coefficients correspond to an incoherent background flow which is homogeneous and does not compress well, neither in a wavelet basis nor in any other basis, and is thus considered as noise.

Different eduction methods to extract coherent structures out of turbulent flows have been proposed for many years. They are based on either one of the following:

(i) clipping, also called 'threshold technique', since one retains only the regions in which the modulus of vorticity is larger than a certain proportion (to be tuned) of the vorticity variance;

(ii) correlation with a template, whose shape (to be adapted) is similar to a typical structure;

(iii) conditional averaging (Antonia 1981), e.g. phase averaging (Hussain 1986) and variable integral time averaging (Blackwelder 1977).

For more details, we refer the reader to several review papers (Cantwell 1981; Fiedler 1988; Robinson 1991) which present the different eduction methods commonly in use. They all require strong hypotheses on the flow, which has to be statistically steady, but also on the coherent structures themselves, since one needs to adjust parameters, e.g. threshold value, template and condition, to extract them. They also present the drawback that the spatial support and intensity of the coherent structures strongly vary depending on the chosen parameters, which unfortunately cannot be estimated a priori. Moreover, the smoothness of the coherent structures is not preserved if discontinuities are introduced, e.g. by clipping, which affect the Fourier spectrum and hence yield an erroneous scaling.

Since there is not yet an universal definition of the coherent structures observed in turbulent flows and to overcome the drawbacks of the previous eduction methods, we change the viewpoint and choose an apophatic approach: instead of defining what coherent structures are, we only define what they are not. For this we propose the minimalist, and hopefully consensual, hypothesis: coherent structures are not noise. Consequently, we get the following definition: coherent structures correspond to what remains after denoising. 
For the noise we use the mathematical definition of randomness, stating that a noise is homogeneously distributed in any functional basis. In other words, a noise by definition cannot be compressed, and its shortest description is itself. Note that not all experimental noises are random; e.g. the $50 \mathrm{~Hz}$ electrical noise from the power supply is periodic and therefore compresses well in the Fourier basis.

This new way of defining coherent structures presents several advantages:

(i) it does not implicitly assume ensemble averaging, as previous definitions do (Hussain 1986), and it works for each flow realization independent of the others;

(ii) it does not require adjustment of parameters, such as threshold value, or template;

(iii) it is quite insensitive to the choice of the wavelet basis, since it is the multiresolution construction of the basis and not the wavelet shape that matters, i.e. wavelets are not used as templates but as a way to organize the data in a scaledependent fashion;

(iv) it tracks coherent structures even when their shape and amplitude change due to, e.g., pairing, stretching or tearing;

(v) it allows to process 'incomplete data'.

Data incompleteness is encountered when the dimensionality of the measured data is lower than the dimensionality of the flow itself, e.g. a two-dimensional cut measured by particle image velocimetry (PIV) in a three-dimensional flow. Note that incompleteness is different from discretization, i.e. sampling (which plays a different role that shall also be taken into account). If the eduction method needs templates of typical coherent structures, it cannot treat incomplete data. Indeed, to define the templates, one should first describe how the probe will see all possible motions and distortion of the coherent structures passing by. Since ours requires a model of the noise but not of the coherent structures themselves (no templates needed), it can treat any data, complete or incomplete, the same way.

Based on the previous principles, we have proposed a new wavelet-based eduction method in Farge et al. (1992), which we have improved in Farge, Schneider \& Kevlahan (1999) using the denoising theorem demonstrated by Donoho \& Johnstone (1994). It decomposes the vorticity field into an orthogonal wavelet basis and separates the wavelet coefficients into two classes: those whose modulus is stronger than a threshold, whose value is automatically adjusted, since it depends only on the enstrophy and the resolution, and those whose modulus is weaker. The coherent vorticity field is reconstructed from the strongest wavelet coefficients by an inverse wavelet transform, while the incoherent vorticity field is obtained by subtracting the coherent from the total vorticity. Both fields being orthogonal, enstrophy has thus been split into coherent and incoherent contributions. The coherent and incoherent velocities are then reconstructed from the coherent and incoherent vorticities by applying the Biot-Savart's kernel.

To demonstrate the advantages of the different wavelet tools presented above, we will apply them to analyse the time evolution of a bursting vortex, chosen as a typical unsteady and inhomogeneous flow which may play a key role to explain the turbulent cascade. We have designed an experimental setting (see figure 1 and movie 1) in which a vortex tube emerges, is then advected and stretched by a steady laminar flow and later becomes unstable and bursts while producing vorticity sheets and filaments which spread all over space. Although the flow rate is maintained steady, the vortex life cycle reproduces quasi-periodically. Using PIV we measure the time evolution of the three components of velocity in a vertical plane perpendicular to the vortex axis and located in the middle of the channel. 


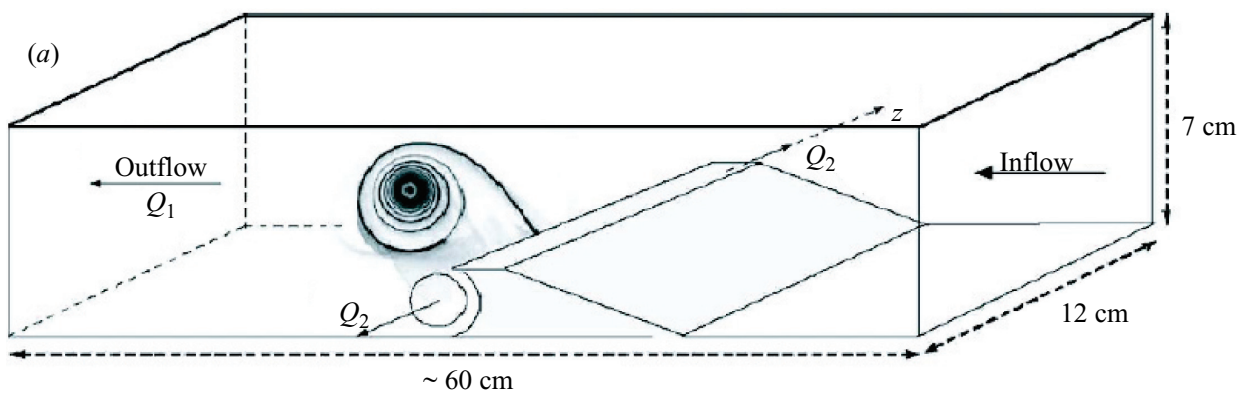

(b)
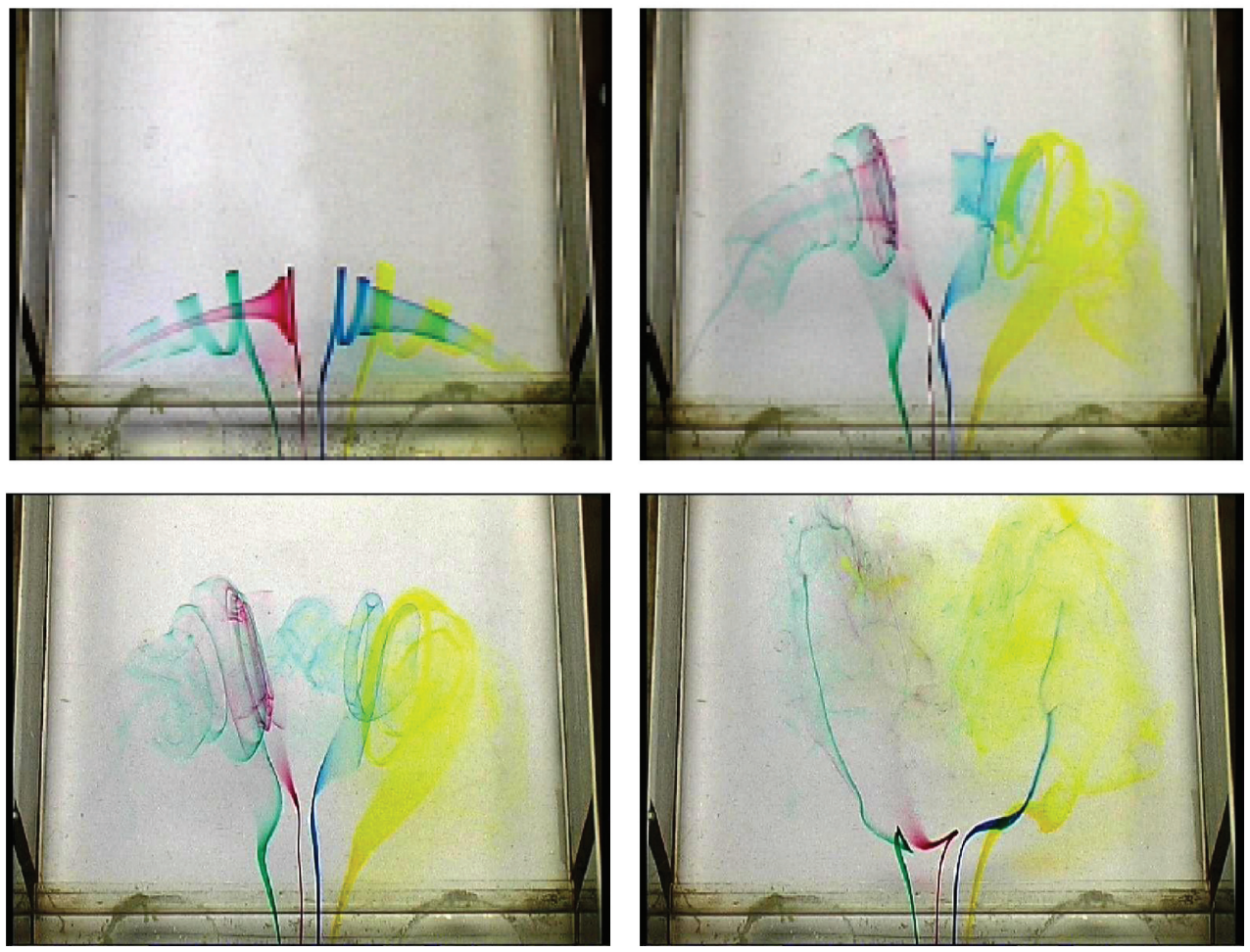

Figure 1. (a) Schematic of the experiment (from Cuypers et al. 2004). The vortex initiated by the step (11 mm high) is strained by the channel flow $Q_{1}$ and intensified and stretched by the axial suction $Q_{2}$; therefore the total flow rate through the channel is $\left(Q_{1}+2 Q_{2}\right)$. The values $Q_{1}=208 \mathrm{~cm}^{3} \mathrm{~s}^{-1}$ and $Q_{2}=125 \mathrm{~cm}^{3} \mathrm{~s}^{-1}$ are chosen to produce a vortex life cycle which quasi-periodically repeats itself. $(b)$ Time evolution of one vortex bursting. Time advances from left to right and top to bottom. Each picture is separated from the next by about $1 \mathrm{~s}$. (See also movie 1 showing the experiment running.)

The paper is organized as follows. We first review the CWT and the OWT. We next describe the wavelet-based statistical tools and techniques for coherent vortex extraction. We then present the experimental setting to study the bursting of a stretched vortex advected by a laminar flow. We discuss the results obtained by applying different wavelet tools. Finally, we draw conclusions and propose perspectives. 


\section{Wavelets}

\subsection{Continuous wavelet transform}

The continuous wavelet transform (CWT) is an integral transform (as is the case of the Fourier transform) which unfolds a field into space, scale and optionally angular contributions. In the following, we consider as example a two-dimensional scalar field of finite energy, i.e. $f(x) \in L^{2}\left(R^{2}\right)$. The CWT uses analysing functions $\psi_{l, x^{\prime}}(\boldsymbol{x})$ which are obtained by continuously translating and dilating a function $\psi(x) \in L^{2}\left(R^{2}\right)$, the 'mother wavelet'. By definition a wavelet is an oscillating function of zero mean that is well localized in both physical space and spectral space. Indeed, to be a wavelet a function $\psi(\boldsymbol{x})$ should verify the admissibility condition

$$
C_{\psi}=\int_{R^{2}}|\widehat{\psi}(\boldsymbol{k})|^{2} \frac{\mathrm{d}^{2} \boldsymbol{k}}{|\boldsymbol{k}|^{2}}<\infty,
$$

where

$$
\widehat{\psi}(\boldsymbol{k})=\int_{R^{2}} \psi(\boldsymbol{x}) \mathrm{e}^{-\mathrm{i} 2 \pi \boldsymbol{k} \cdot \boldsymbol{x}} \mathrm{d} \boldsymbol{x} .
$$

From the mother wavelet $\psi(x)$ we generate the family of translated, rotated and dilated wavelets

$$
\psi_{l, x^{\prime}, \theta}(\boldsymbol{x})=l^{-1} \psi\left[\Omega^{-1}(\theta) \frac{\boldsymbol{x}-\boldsymbol{x}^{\prime}}{l}\right],
$$

where $l \in R^{+}$is the scale of the analysing wavelet, $\boldsymbol{x}^{\prime} \in R^{2}$ its position, and $\theta \in[0,2 \pi[$ its orientation with $\Omega(\theta) \in\left(R^{2} \times R^{2}\right)$ the unitary rotation tensor,

$$
\Omega(\theta)=\left(\begin{array}{cc}
\cos \theta & -\sin \theta \\
\sin \theta & \cos \theta
\end{array}\right)
$$

We can thus study the flow anisotropy by varying the wavelet orientation, which is optional but only possible if we choose an anisotropic wavelet, such as the Morlet wavelet. If we do not want to distinguish directions, there is always the possibility to average the wavelet coefficients over angles.

Each wavelet coefficient $\widetilde{f}\left(l, \boldsymbol{x}^{\prime}\right)$, indexed by position $\boldsymbol{x}^{\prime}$, direction $\theta$ and scale $l$, is given by

$$
\tilde{f}\left(l, \boldsymbol{x}^{\prime}, \theta\right)=\left\langle f(\boldsymbol{x}), \psi_{l, \boldsymbol{x}^{\prime}, \theta}\right\rangle=\int_{R^{2}} f(\boldsymbol{x}) \psi_{l, \boldsymbol{x}^{\prime}, \theta}^{*}(\boldsymbol{x}) \mathrm{d}^{2} \boldsymbol{x},
$$

where $\langle.,$.$\rangle denotes the L^{2}$ inner product and * denotes the complex conjugate.

Because the wavelet transform is invertible, the field $f(x)$ can be recovered from its wavelet coefficients $\widetilde{f}\left(l, \boldsymbol{x}^{\prime}\right)$ according to

$$
f(\boldsymbol{x})=C_{\psi}^{-1} \int_{R^{2}} \int_{R^{+}} \int_{0}^{2 \pi} \widetilde{f}\left(l, \boldsymbol{x}^{\prime}, \theta\right) \psi_{l, \boldsymbol{x}^{\prime}, \theta}(\boldsymbol{x}) \frac{\mathrm{d} l \mathrm{~d} \Omega(\theta) \mathrm{d}^{2} \boldsymbol{x}^{\prime}}{l^{3}},
$$

where $C_{\psi}$ is given by the admissibility condition (2.1), and its finite value insures invertibility of the wavelet transform.

Since the wavelet transform is integral, it conserves energy (Parseval's relation) in the following manner:

$$
E=\int_{R^{2}}|f(\boldsymbol{x})|^{2} \mathrm{~d}^{2} \boldsymbol{x}=C_{\psi}^{-1} \int_{R^{2}} \int_{R^{+}} \int_{0}^{2 \pi}\left|\tilde{f}\left(l, \boldsymbol{x}^{\prime}, \theta\right)\right|^{2} \frac{\mathrm{d} l \mathrm{~d} \Omega(\theta) \mathrm{d}^{2} \boldsymbol{x}^{\prime}}{l^{3}} .
$$




\subsection{Choice of the analysing wavelet}

The analysing wavelet can be either real valued, e.g. Mexican hat, or complex valued, e.g. Morlet wavelet. Complex wavelets are preferred for analysis purposes, since, as is the case for the Fourier transform, one can separately study the modulus and the phase of the resulting complex-valued coefficients. This is the reason why in the present paper we will use as analysing wavelet the two-dimensional complex Morlet wavelet, which is a plane wave modulated by a Gaussian envelope defined as

$$
\psi(\boldsymbol{x})=\mathrm{e}^{\mathrm{i} \boldsymbol{k}_{\psi} \cdot \boldsymbol{x}} \mathrm{e}^{-\frac{1}{2}|x|^{2}},
$$

where $k_{\psi}$ is the centroid wavenumber of the mother wavelet, i.e. the barycenter of its support in spectral space, defined as

$$
k_{\psi}=\frac{\int_{R^{2}}|\boldsymbol{k}||\hat{\psi}(\boldsymbol{k})| \mathrm{d}^{2} \boldsymbol{k}}{\int_{R^{2}}|\hat{\psi}(\boldsymbol{k})| \mathrm{d}^{2} \boldsymbol{k}} .
$$

The Morlet wavelet is only marginally admissible and $k_{\psi}$ should be large enough to satisfy the zero-mean criterion (2.1). In this paper we choose $k_{\psi}=6$, which guarantees that the mean of the wavelet is zero up to machine round-off error.

We sample directions on 12 angles denoted by $\theta$ and distributed uniformly from 0 to $\pi / 2$. We then average the wavelet coefficients over them to recover an isotropic analysis, since we focus here only on the space and scale contributions of the flow.

For the numerical implementation of the CWT we calculate the coefficients, for a given scale and orientation, by convolving the field with the corresponding wavelet. In order to simplify the computation we assume the function to be periodic. We can then compute the reconstruction formula (2.6) directly in spectral space,

$$
f(\boldsymbol{x})=C_{\psi}^{-1} \int_{R^{2}} \int_{R^{+}} \int_{0}^{2 \pi} \widehat{f}\left(l, \boldsymbol{k}^{\prime}, \theta\right) \widehat{\psi}_{l, \boldsymbol{k}, \theta}\left(\boldsymbol{k}^{\prime}\right) \mathrm{e}^{\mathrm{i} \boldsymbol{k}^{\prime} \cdot \boldsymbol{x}} \frac{\mathrm{d} \Omega(\theta) \mathrm{d} l \mathrm{~d}^{2} \boldsymbol{k}^{\prime}}{l^{3}} .
$$

Using the fast Fourier transform (FFT), the operation count is $\left(3 N_{x} \log _{2} N_{x}+\right.$ $\left.N_{x}\right) N_{\theta} N_{l}$, where $N_{x}$ is number of space samples, $N_{l}$ the number of analysed scales and $N_{\theta}$ the number of analysed directions. This requires more operations than the FFT itself $\left(N_{x} \log _{2} N_{x}\right)$; however, the operation count is reduced to order- $N$ operations if one uses compactly supported orthogonal wavelets, as explained below.

\subsection{Orthogonal wavelet transform}

For the CWT the analysing wavelets are continuously translated, rotated and dilated; therefore the continuous wavelet representation is redundant, since we started with a field $f(x) \in L^{2}\left(R^{2}\right)$ in physical space of two dimensions and obtained coefficients $\widetilde{f}\left(l, \boldsymbol{x}^{\prime}, \theta\right)$ in wavelet space of four dimensions, or three dimensions after averaging over angles. This results in a correlation between neighbouring wavelet coefficients, which may bias the interpretation of the correlation inherent to the field itself. It is possible to overcome this by using an orthogonal wavelet transform (OWT) that eliminates the correlation between the wavelet coefficients. Such a transform is based upon a multiresolution analysis (MRA), which gives successive approximations of the field at different resolutions, from the smallest scale (twice the grid size) to the largest scale (the domain size). The wavelet coefficients at a given scale contain the information necessary to go from one approximation to the next, since they encode the difference between successive scales. An additional function, called the scaling function, $\phi(x) \in L^{2}\left(R^{2}\right)$, whose average is normalized to one, is introduced to encode information about the approximations to the field at different scales. Thus the 
Fourier-transformed wavelets $\hat{\psi}$ act as a set of high-pass filters, and the Fouriertransformed associated scaling functions $\hat{\phi}$ as a complementary set of low-pass filters. The scaling and wavelet coefficients give at each scale the mean and fluctuating components, respectively.

To guarantee orthogonality a particular sampling of the wavelet space is required. It corresponds to the dyadic grid, i.e. the scale axis is discretized by octaves, while the space axis is discretized by discrete translation steps whose size varies with the scale. For a function of support $L$ sampled at $N=2^{J}$ points, the total number of discrete scales (or octaves) is $J$, the number of wavelet coefficients $\widetilde{f}_{j i}$ at each scale is $2^{j-1}$, and their spacing on the dyadic grid is $2^{-j} i$, where $i$ and $j$ are position and scale indices, respectively. The scales run from twice the size of the grid, $l_{\min }=2^{-(J-1)} L$, to the entire domain size, $l_{\max }=2^{0} L$.

Note that usually one uses compactly supported wavelets (as is the case in this paper), meaning that wavelets are non-zero valued over a finite interval, which doubles for each successively larger scale. However, each wavelet on the dyadic grid is orthogonal to the other wavelets, i.e. $\left\langle\psi_{j, i}, \psi_{j^{\prime}, i^{\prime}}\right\rangle=\delta_{j, j^{\prime}} \delta_{i, i^{\prime}}$, where $j, j^{\prime}$ and $i, i^{\prime}$ are scale and position indices, respectively.

The orthogonal wavelets in two dimensions are obtained by tensor product of two one-dimensional orthogonal wavelets. Therefore there are three wavelets corresponding to three directions indexed by $\mu$, defined as

$$
\psi_{j, i_{x}, i_{y}}^{\mu}= \begin{cases}\psi_{j, i_{x}} \times \phi_{j, i_{y}} & \text { for } \mu=1 \text { (horizontal), } \\ \phi_{j, i_{x}} \times \psi_{j, i_{y}} & \text { for } \mu=2 \text { (vertical), } \\ \psi_{j, i_{x}} \times \psi_{j, i_{y}} & \text { for } \mu=3 \text { (diagonal), }\end{cases}
$$

and the associated scaling function

$$
\phi_{j, i_{x}, i_{y}}=\phi_{j, i_{x}} \times \phi_{j, i_{y}},
$$

where $i_{x} \in\left[0,2^{J-1}-1\right]$ and $i_{y} \in\left[0,2^{J-1}-1\right]$ are the $x$ and $y$ positions of the index.

The two-dimensional field $f(\boldsymbol{x})$ is projected on to the orthogonal wavelet basis $\left[\psi_{j, i_{x}, i_{y}}^{\mu}\right]$ as

$$
f(\boldsymbol{x})=\phi_{0,0,0} \bar{f}_{0,0,0}+\sum_{j=0}^{J-1} \sum_{\mu=1}^{3} \sum_{i_{x}, i_{y}=0}^{2^{j-1}-1} \widetilde{f}_{j, i_{x}, i_{y}}^{\mu} \psi_{j, i_{x}, i_{y}}^{\mu},
$$

where the sum is over the scales indexed by $j$, positions indexed by $\left(i_{x}, i_{y}\right)$ and directions indexed by $\mu$. The scaling coefficient at the largest scale $\bar{f}_{0,0,0}$ corresponds to the mean of the field. The wavelet coefficients are given by

$$
\widetilde{f}_{j, i_{x}, i_{y}}^{\mu}=\left\langle f(\boldsymbol{x}), \psi_{j, i_{x}, i_{y}}^{\mu}\right\rangle,
$$

where $\langle.,$.$\rangle denotes the L^{2}$ inner product in two dimensions, defined as

$$
\left\langle f_{i}, f_{j}\right\rangle=\int_{R^{2}} f_{i}(\boldsymbol{x}) \cdot f_{j}(\boldsymbol{x}) \mathrm{d}^{2} \boldsymbol{x} .
$$

Due to the orthogonality of the basis, a field sampled on $N$ points is represented by $N$ wavelet coefficients. The compactly supported wavelets we use in this study are defined by two quadrature mirror filters of finite length $C$, and the wavelet coefficients are computed by convolving the field with the filter coefficients. This corresponds to the fast wavelet algorithm (Mallat 1989) for the transform and its inverse, whose 
computational cost is $C N$ operations. The OWT is significantly faster to compute than the CWT and the FFT, whose computational cost is $N \log _{2} N$ operations. Similar to the FFT, the fast OWT requires the sampling to be a power of 2, i.e. $N=2^{J}$. We choose here the Coifman 12 wavelet which has its first four moments as zero and corresponds to a filter of length $C=12$.

For a review of the different wavelet transforms and their applications to turbulence, see Farge (1992). For turbulence analysis using orthogonal wavelets, see Meneveau (1991). Related papers can be downloaded from the 'Publications' section at http://wavelets.ens.fr.

\section{Wavelet tools}

\subsection{Wavelet spectrum}

As explained in Farge (1992), for data analysis it is better to use a complex wavelet, since the modulus of the complex-valued wavelet coefficients gives the energy density, in a fashion similar to Fourier analysis. In this paper we analyse a two-dimensional cut of velocity field $\boldsymbol{v}(\boldsymbol{x})$ measured by PIV. We consider a window of size $N=128 \times 128$, which gives $J=7$ octaves, and compute the wavelet coefficients for 12 different directions using a two-dimensional Morlet wavelet. Since we are more interested in the space-scale properties of the flow than its eventual anisotropy, we then average the modulus of the wavelet coefficients over the 12 directions to get $|\widetilde{v}(l, \boldsymbol{x})|$, from which we compute the space-scale energy density

$$
E(l, x)=\frac{|\widetilde{v}(l, x)|^{2}}{l^{2}} .
$$

Similarly, the square modulus of the CWT coefficients of the vorticity field, i.e. the curl of velocity, yields the space-scale enstrophy density $Z(l, x)$.

The space-scale energy density (3.1) can then be integrated over all scales to obtain the local energy density

$$
E(\boldsymbol{x})=C_{\psi}^{-2} \int_{0^{+}}^{\infty} E(l, x) \frac{\mathrm{d} l}{l} .
$$

In an alternative way, the space-scale energy density (3.1) can be integrated over space to obtain the scalogram $E(l)$, which gives the energy density as a function of scale:

$$
E(l)=\int_{R^{2}} E(l, \boldsymbol{x}) \mathrm{d}^{2} \boldsymbol{x} .
$$

Since scale is related to wavenumber by the relation $l=k_{\psi} / k$, where $k_{\psi}$ is the centroid wavenumber of the wavelet as defined in (2.9), the scalogram is related to the Fourier energy spectrum $E(\boldsymbol{k})=|\widehat{v}(\boldsymbol{k})|^{2}$, also called spectrogram, according to

$$
E(l)=\int_{R^{2}} E(\boldsymbol{k})|\hat{\psi}(\boldsymbol{k})|^{2} \mathrm{~d}^{2} \boldsymbol{k} .
$$

The scalogram thus corresponds to the spectrogram $E(\boldsymbol{k})$ smoothed by the Fourier transform of the analysing wavelet (Farge 1992). The total energy is recovered by integrating over all scales:

$$
E=C_{\psi}^{-2} \int_{0^{+}}^{\infty} E(l) \frac{\mathrm{d} l}{l} .
$$




\subsection{Intermittency measure}

To quantify the flow intermittency we have proposed in Farge et al. (1990) the LIM. It corresponds to the spatial distribution of the local deviation from the wavelet energy spectrum at a given scale and is defined as

$$
I(l, x)=\frac{|\widetilde{v}(l, x)|^{2}}{\int_{R^{2}}|\widetilde{v}(l, x)|^{2} \mathrm{~d}^{2} x} .
$$

If everywhere $I(l, \boldsymbol{x}) \sim 1$, at each scale the energy is distributed evenly in space and the flow is non-intermittent, as is the case, e.g., for one realization of a Gaussian white noise. In contrast, if locally $I(l, x) \gg 1$, the flow presents spotty features, such as coherent structures, and is intermittent.

We can then define the global intermittency measure (GIM) by integrating in space and scale the squared LIM:

$$
I=\int_{R^{2}} \int_{0^{+}}^{\infty}|I(l, \boldsymbol{x})|^{2} \frac{\mathrm{d} l}{l} \mathrm{~d}^{2} \boldsymbol{x} .
$$

\subsection{Extraction of coherent structures}

Since there is not yet any agreement among specialists of turbulence about what coherent structures are and which method is the best to extract them, we propose an apophatic approach in which, rather than stating what the coherent structures are, we make assumptions about what they are not. We suppose that 'coherent structures are different from noise', and thus we define them as 'what remains after denoising'. To get started we choose the simplest hypothesis for the noise, namely that it is additive, Gaussian and white, i.e. decorrelated.

In Farge et al. $(1992,1999)$ we proposed to extract coherent structures by filtering the orthogonal wavelet coefficients of the vorticity field: the coefficients whose modulus is larger than a threshold $\epsilon$ reconstruct the coherent component, while the remaining coefficients correspond to the incoherent component. It has been demonstrated in Donoho \& Johnstone (1994) that the optimal threshold, in a min-max sense, to remove an additive Gaussian white noise is

$$
\epsilon=\sqrt{2 \sigma_{I}^{2} \log _{e} N}
$$

where $\sigma_{I}^{2}$ is the variance of the noise and $N$ the resolution at which the field has been sampled. This value ensures a vanishing probability that the wavelet coefficients of the noise have a value larger than the threshold.

Since the variance of the noise we would like to remove is not known a priori, we have developed in Azzalini, Farge \& Schneider (2005) a recursive algorithm to estimate the variance of the noise from the variance of the incoherent wavelet coefficients.

The algorithm consists of three steps that we describe below.

(a) Initialization

(i) Calculate the OWT of the vorticity field $\omega$ to obtain its wavelet coefficients $\widetilde{\omega}$.

(ii) Take the variance of the total vorticity field, as a first estimate for the variance of the noise, as follows:

$$
\sigma_{0}^{2}=1 / N \sum|\omega(\boldsymbol{x})|^{2}=2 Z
$$

where $Z$ is the total enstrophy. 
(iii) Compute the threshold $\epsilon_{0}=\sqrt{2 \sigma_{0}^{2} \log _{2} N}$ based upon this first estimate of the noise variance.

(iv) Set the loop count $n=0$.

(b) Iteration loop

(i) Compute a new estimate of the variance $\sigma_{n+1}^{2}=1 / N \sum\left|\widetilde{\omega_{I}}\right|^{2}$ of the incoherent vorticity from the incoherent wavelet coefficients $\widetilde{\omega}_{I}$ whose modulus is smaller than the threshold $\epsilon_{n}$, where

$$
\widetilde{\omega}_{j, i_{x}, i_{y}}^{\mu, I}= \begin{cases}\widetilde{\omega}_{j, i_{x}, i_{y}}^{\mu} & \text { if }\left|\widetilde{\omega}_{j, i_{x}, i_{y}}^{\mu}\right| \leqslant \epsilon_{n}, \\ 0 & \text { otherwise. }\end{cases}
$$

(ii) Compute the new threshold $\epsilon_{n+1}=\sqrt{2 \sigma_{n+1}^{2} \log _{e} N}$.

(iii) Update loop count $n=n+1$.

(iv) Count the number of incoherent coefficients $N_{I}^{n}$ whose modulus is smaller than the threshold $\epsilon_{n}$.

(v) If the number of coefficients $N_{I}^{n+1}$ is the same as $N_{I}^{n}$, then proceed to reconstruction, else repeat the loop until the number of incoherent coefficients converges.

(c) Reconstruction

(i) Reconstruct the coherent vorticity in physical space from the coherent wavelet coefficients whose modulus is above the threshold using the inverse OWT:

$$
\widetilde{\omega}_{j, i_{x}, i_{y}}^{\mu, C}= \begin{cases}\widetilde{\omega}_{j, i_{x}, i_{y}}^{\mu} & \text { if }\left|\widetilde{\omega}_{j, i_{x}, i_{y}}^{\mu}\right| \geqslant \epsilon_{n}, \\ 0 & \text { otherwise. }\end{cases}
$$

(ii) Compute the incoherent component in physical space by subtracting the coherent component from the total field $\omega_{I}(\boldsymbol{x})=\omega(\boldsymbol{x})-\omega_{C}(\boldsymbol{x})$.

Due to the orthogonality of the wavelet transform, the variance $\sigma^{2}$ is partitioned among the components, i.e. $\sigma^{2}=\sigma_{C}^{2}+\sigma_{I}^{2}$.

The operation count of the algorithm is essentially determined by the two OWTs (one forward, one inverse), each requiring order- $N$ operations. We have proved in Azzalini et al. (2005) that this recursive algorithm for automatically estimating the threshold converges. The convergence rate actually depends upon the signal-to-noise ratio between the coherent and incoherent components, which we call the coherency measure $(\mathrm{CM})$, defined as

$$
C M=10 \log _{10}\left(\frac{\sigma_{C}^{2}}{\sigma_{I}^{2}}\right) .
$$

As shown in Azzalini et al. (2005), the lower the signal-to-noise ratio (i.e. the stronger the noise), the faster the convergence. For instance if one considers one realization of a noise sampled on $N$ points, the algorithm considers the entire signal as incoherent after only one iteration. Conversely, for a signal uncorrupted by noise the algorithm retains the entire signal as coherent, but after much more (but less than $N$ ) iterations. In practice, only very few iterations are sufficient.

Note that the method proposed here to extract coherent structures out of turbulent flows is very different from the eduction method presented in Berkooz, Holmes \& Lumley (1993), which is based on proper orthonormal decomposition (POD). POD, also called principal component analysis (PCA), empirical orthogonal function (EOF) and Karhunen-Loève decomposition depending on its domain of application, computes the two-point correlation tensor of an ensemble of realizations and then 
diagonalizes it and retains only the eigenmodes having the largest eigenvalues. This yields the best basis for this ensemble of realizations with respect to the $L^{2}$ norm. The retained modes are defined a posteriori for all realizations, as those containing, on average, the most variance. Thus, the eduction procedure based on POD is linear, as the selection of the retained modes does not depend on the realization itself. In contrast, the wavelet-based eduction selects the modes a priori, as those having the strongest amplitudes among all wavelet basis functions. Hence, the selection procedure is nonlinear, as the retained basis functions depend on each flow realization. It can then be applied to successive realizations. The POD preserves the second-order statistics, while the wavelet-based eduction method also respects the higher-order statistics of the flow, which is not the case for POD. A detailed comparison between POD and wavelet eduction methods has been performed in Farge, Pellegrino \& Schneider (2003).

\section{Vortex bursting}

\subsection{Flow under study}

Recent experimental studies have focused on an isolated bursting vortex as a source of turbulence, leading to a transient build-up of turbulent energy cascade (Cuypers, Maurel \& Petitjeans 2003, 2004, 2006). The scaling of the energy spectrum was found to vary from $k^{-1}$ to $k^{-2}$ during the bursting, although the $k^{-5 / 3}$ scaling is recovered in the time-averaged spectrum. The vortex was well approximated by a stretched spiral vortex following the model of Lundgren (1982), which also predicts a $k^{-5 / 3}$ time-averaged energy spectrum. However, the time evolution of the energy spectrum is not well understood, and this is a problem we would like to study in this paper with the help of the wavelet representation.

Previous studies were conducted using hot-film anemometry (Cuypers et al. 2003, 2004), which has a good time resolution but requires a local Taylor hypothesis to obtain the spatial information necessary to calculate the energy spectrum. More recently, PIV was used to measure the spatial distribution of the velocity field directly, without inferring it from a time series, which would have required Taylor's hypothesis. Simultaneous hot-film probe measurements were used to synchronize the phase of the PIV with the bursting of the vortex. The PIV measurements were then phase averaged to obtain an ensemble average, from which was computed the average time record of the bursting.

The scaling of the energy spectrum in the inertial range, obtained from the PIV measurements, was in good agreement with the previous hot-film measurements (Cuypers et al. 2006). However, the time resolution of the measurements was too low for a single burst to be properly analysed in time. In the current study we use higher time-resolution PIV to track the time evolution of each individual bursts. This allows us to free ourselves from the phase-averaging assumption and to directly study the transient behaviour of the flow.

\subsection{Experimental setting}

The experimental test section is a long channel of $12 \times 7 \mathrm{~cm}$ rectangular cross-section, shown in figure $1(a)$. The walls are translucent Plexiglas in order to easily observe the flow. The channel is filled with water, and the flow rate $Q_{1}$ down the channel is regulated. The mean flow velocity is between 0 and $10 \mathrm{~cm} \mathrm{~s}^{-1}$, and the initial flow is laminar. At the bottom of the test section is an $11 \mathrm{~mm}$ step that initiates the vortex. Two holes have been made in the transverse channel walls at the location of the step at which some water is pumped out at a controlled flow rate $Q_{2}$. This suction 
produces a stretching of the initial vortex which strongly intensifies and concentrates its vorticity. This also pins the edges of the vortex to the suction holes in the channel walls. Thus, a strong stretched vortex is produced in a laminar flow downstream the step at which the flow separates.

The total flow rate through the channel is $\left(Q_{1}+2 Q_{2}\right)$. If $Q_{1}$ is sufficiently small, the vortex remains stable once it appears and persists during the whole experiment with no change in either size or intensity. As $Q_{1}$ increases, the vortex is carried further downstream the step by the mean channel flow. It is then bent as long as it remains pinned at the sidewalls. Above a critical channel flow rate the vortex detaches from the suction holes and bursts. Notice that the production of turbulence is only due to the vortex bursting, since the flow was previously laminar. Turbulence then decays before being carried out of the observation window by the mean flow $Q_{1}$. Another vortex is then produced which exhibits the same dynamics. This new vortex is also produced in a laminar flow which has recovered from the previous vortex bursting. The same cycle repeats itself quasi-periodically at intervals of approximately 6-12 s depending on the flow rate $Q_{1}$. The values $Q_{1}=208 \mathrm{~cm}^{3} \mathrm{~s}^{-1}$ and $Q_{2}=125 \mathrm{~cm}^{3} \mathrm{~s}^{-1}$ for the present experiment were chosen to produce the same kind of intense bursting vortex as used in previous works, e.g. Cuypers et al. (2004). The Reynolds number based upon the circulation of the vortex is estimated to be about 4000 . We visualize the vortex, either by injecting dye into the channel or by seeding the flow with tracer particles to perform PIV. Streams of dye injected upstream the step are engulfed into the vortex, and one can then observe the vortex life cycle as illustrated in figure $1(b)$ (see also movies $1 a$ and $1 b$ ).

The PIV measurements are taken in a vertical plane perpendicular to the vortex axis located in the middle of the channel. Digital images are acquired at 15 double frames per second with a resolution of $1600 \times 1200$ pixels. We use a pulsed laser to obtain exposures of successive image frames each $1 \mathrm{~ms}$ using frame straddling. We then apply standard PIV algorithms on image pairs to measure the velocity field at a rate of $15 \mathrm{~Hz}$ in a $6.4 \times 4.8 \mathrm{~cm}$ observation window. This zone is sampled with $200 \times 150$ vectors which correspond to a spatial resolution of a vector every $0.032 \mathrm{~cm}$. The cross-correlation function is computed by using multi-pass refining on windows of sizes $64 \times 64$ pixels to $16 \times 16$ pixels with $50 \%$ overlap. All these steps have been performed using Davis 7.2 software.

The size of the PIV observation window results from a compromise between sufficient spatial resolution and vortex tracking while it bursts and moves downstream the channel. The vorticity component perpendicular to the plane is calculated from the measured two-dimensional velocity field. The PIV acquisition is performed during several vortex life cycles. Each experimental run generates 345 snapshots corresponding to $23 \mathrm{~s}$ of the flow evolution, which is the maximum allowed by the memory of the camera.

\subsection{The measured flow}

The time evolution of a single vortex life cycle as seen in the observation plane is shown in figure 2 . The modulus of velocity field and the vorticity perpendicular to the observation plane are shown in figures $2(a)$ and $2(c)$, respectively.

A time trace of the bursting over three vortex life cycles is shown in figure $2(b)$, which agrees well with the previous hot-film measurements of velocity. We observe that the velocity increases when the vortex approaches the observation plane; it then peaks just before the vortex begins to burst; the velocity then rapidly decreases, although it remains highly fluctuating. After the vortex has burst, its remnants are swept down the channel before another vortex is formed, thus repeating the cycle. 

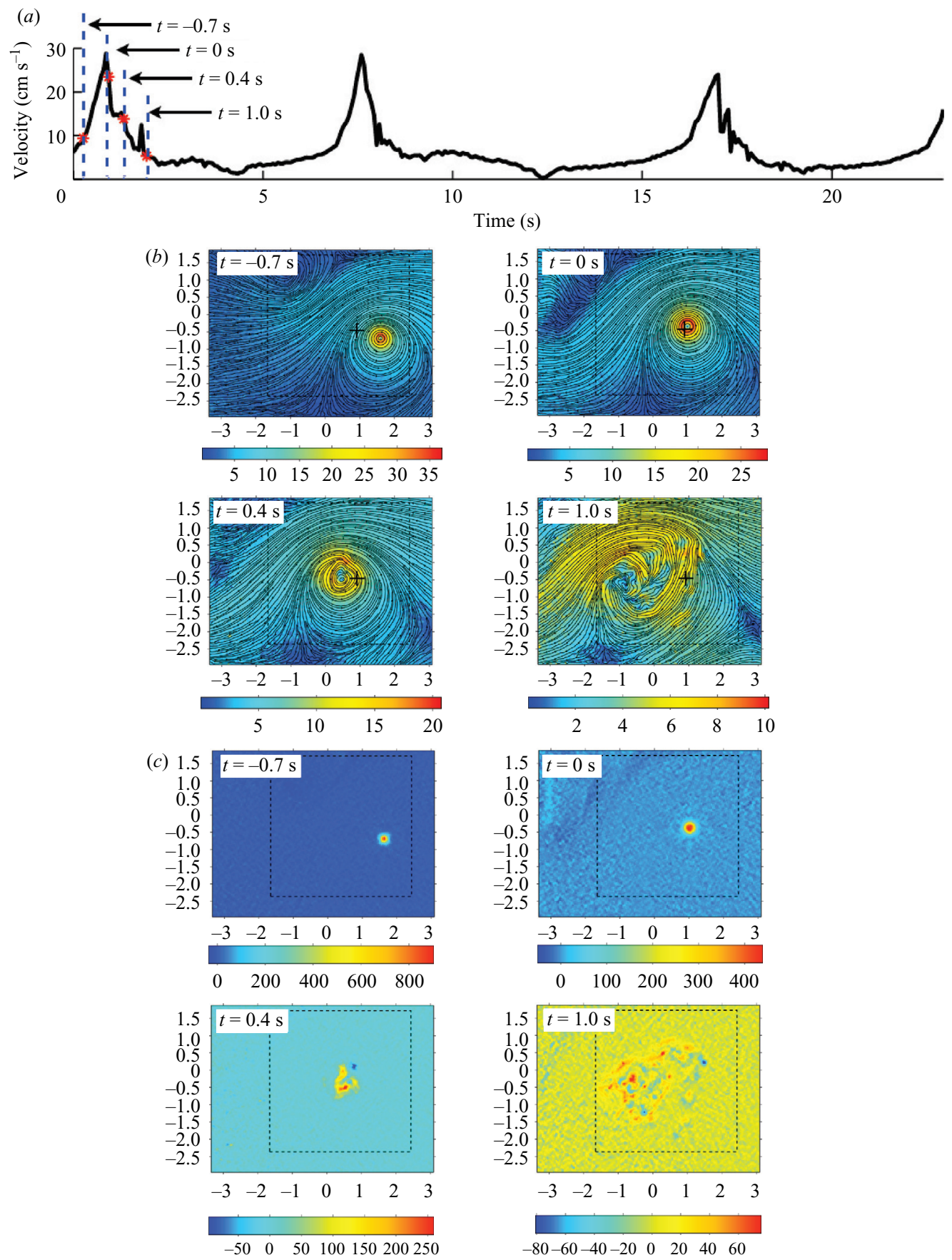

Figure 2. Time evolution of the vortex bursting. (a) Time trace of the modulus of velocity measured in one location (indicated by a cross on the velocity field below) and averaged in a $3 \times 3$ region around that point. The dashed vertical lines indicate the instants $t=\{-0.7,0,0.4,1.0\} \mathrm{s}$ corresponding to the snapshots below, with $t=0$ the beginning of the bursting. ( $b$ ) Modulus of velocity with streamlines superimposed. The channel flow moves from right to left. (c) Modulus of vorticity. Notice that for both fields only the component perpendicular to the observation plane is shown. (See also movie $2 a$ showing the time evolution of the velocity modulus, movie $2 b$ showing the time evolution of the vorticity modulus and movie $2 c$ showing the time evolution of the energy spectrum.) 
However, with the previous hot-film measurements the location at which we took measurements was fixed in the channel, off the plane chosen for the PIV-measurement acquisition. Actually, the vortex does not always burst at the same location or appear with the same intensity. Therefore a peak in the trace of the velocity modulus, either measured by hot film or at a fixed plane by PIV, does not necessarily indicate that the vortex has burst at that location and at that instant. Due to the good time resolution of the PIV data and using a technique presented by Cuypers et al. (2004), we can determine the precise instant when the vortex starts bursting. It has been shown in Cuypers et al. (2004) that, for the same experimental parameters as those chosen here, the time needed to build the energy cascade is approximately $1.5 \mathrm{~s}$. Before bursting the vortex has a quasi-Gaussian shape, which is consistent with the expected profile along the mid-plane of the channel. The background flow of the channel, about $1 \mathrm{~cm} \mathrm{~s}^{-1}$, is of relatively low intensity compared to that of the largest values measured in the vortex, up to $40 \mathrm{~cm} \mathrm{~s}^{-1}$. We observe a slight asymmetry of the velocity field, which is due to the additional background flow, although until it bursts, the vortex remains axisymmetric.

The vortex bursting is hard to analyse due to its highly non-stationary behaviour. To overcome this difficulty, we have selected four characteristic instants $t=\left\{t_{1}, t_{2}, t_{3}, t_{4}\right\}$ defined as follows:

(i) $t_{1}=-0.7 \mathrm{~s}$, just after the formation of the vortex when it is still stable;

(ii) $t_{2}=0.0 \mathrm{~s}$, the beginning of the bursting;

(iii) $t_{3}=0.4 \mathrm{~s}$, after the vortex has broken down into pieces;

(iv) $t_{4}=1.0 \mathrm{~s}$, when the remaining pieces have dissipated.

Since our observations are in a two-dimensional plane taken from a threedimensional field, only the first two stages of the flow, when the vortex is still stable and quasi-two-dimensional, can be interpreted without too much ambiguity. This is no more the case for the following stages, $t_{3}$ and $t_{4}$, when the flow has become fully three-dimensional and remnants of the burst vortex appear and disappear from the observation plane.

\section{Results: extraction of coherent structures}

\subsection{Vorticity evolution}

We consider the evolution of the velocity (see movie $2 a$ ), and from it we compute the vorticity field at each time step. (The vorticity evolution is shown in movie $2 b$.) In figures $2(a)$ and $2(b)$ we focus on the four characteristic instants, $t=\left\{t_{1}, t_{2}, t_{3}, t_{4}\right\}$, defined in $\S 4.3$ and shown in figure 2 . Before bursting, at time $t_{1}=-0.7 \mathrm{~s}$, the vortex is intense and well localized. At the instant when the bursting starts, time $t_{2}=0 \mathrm{~s}$, the spatial support of the vortex spreads, and its intensity is sharply reduced. During the bursting, at time $t_{3}=0.4 \mathrm{~s}$, the vortex has been broken into several weaker structures which continue to spread in space. After the bursting, at time $t_{4}=1 \mathrm{~s}$, those structures begin to nonlinearly interact while being swept away from the observation window. We then use the technique described in $\S 3.3$ to split the vorticity field into two orthogonal, coherent and incoherent, components. Since the decomposition is based on orthogonal wavelets we need the sampling to be factorized in a power of two, and therefore we apply the extraction to a window of $N=128^{2}$ grid-point samples for each vorticity snapshot. The location of the window is shown in figures $2(b)$ and $2(c)$. We also compute the evolution of the energy spectrum (see movie $2 c$ ), whose slope varies from -2 , before the bursting, to -1 , after the bursting. Note that the $k^{-5 / 3}$ scaling, predicted by the statistical theory of homogeneous isotropic turbulence 
in three dimensions (Kolmogorov 1941), is only obtained after time averaging, as previously observed in Cuypers et al. (2003, 2004, 2006).

We then split the vorticity field into coherent and incoherent contributions at each instant, as shown in figure 3. We observe that the coherent vorticity evolution in figure $3(b)$ is very similar to the total vorticity evolution in figure $3(a)$, both being highly non-stationary and presenting the same coherent structures (see movies $2 a$ and $2 b$ ). In contrast to the coherent vorticity, the incoherent component, as observed in figure $3(c)$, remains stationary and homogeneous all along the bursting process. Looking at the time evolution of the incoherent vorticity (see movie $3 c$ ), we notice a small region, located where the coherent vortex is, whose level of fluctuation is slightly higher than the experimental noise level. This is also observed in the evolution of the vorticity cuts, as shown in figure $3(d)$, and may correspond to some incoherent enstrophy associated with the turbulent dissipation produced in the shear layer which develops around the vortex, as proposed in Farge, Pellegrino \& Schneider (2001). This dissipation is due to the fact that the vortex is continuously stretched by the axial suction applied at the outlets, which acts as an external forcing. This forcing is then balanced by the dissipation due to the strong velocity gradients which develop in the shear layer around the vortex. This three-dimensional shear layer can be decomposed in the three directions of a referential attached to the vortex: an axial direction parallel to the stretching, a radial direction associated with the straining produced by the radial dependence of the stretching and an azimuthal direction. The slight augmentation of the incoherent vorticity fluctuations we have noticed (in the observation plane perpendicular to the vortex axis) may be due to both axial and radial dissipation. Note that there is no dissipation in the azimuthal direction, since the vortex is axisymmetric.

In order to quantify the level of turbulent dissipation due to the vortex, we subtract the experimental noise from the incoherent vorticity. For this we estimate the level of experimental noise by averaging the value of the incoherent enstrophy far from the vortex. We then consider a window tracking the vortex, and we subtract the experimental noise level from the incoherent enstrophy. The difference corresponds to the turbulent dissipation, which is actually very weak. It results from the straining of the incoherent background flow by the vortex.

\subsection{Vorticity statistics}

The time evolution of the vorticity variance (second moment of vorticity that is twice the enstrophy) of the total, coherent and incoherent flows, together with the ratio of the vorticity moments such as skewness and flatness, are shown in figures $4(a), 4(b)$ and $4(c)$, respectively (see also movies $4 a$ and $4 b$ ). At the beginning, the vorticity field contains a single vortex and is characterized by large values of enstrophy, skewness and flatness. During the bursting these quantities strongly decrease as the vortex loses its coherence and breaks up. Later the vorticity moments return to large values when a new vortex appears in the observation window. Note that the abrupt increase in vorticity skewness and flatness is an artefact due to the entrance of a new vortex into the observation domain (when only a portion of it is in view). For the three diagnostics, we observe that the coherent flow exhibits the same non-stationary behaviour as the total flow, while the incoherent background flow remains stationary. This confirms the observations made in the previous paragraph.

\subsection{Compression ratio}

In figure $4(d)$ we show that only a small percentage of the wavelet coefficients is sufficient to extract the coherent vorticity, corresponding to the vortex before it bursts and to the remaining structures resulting from its bursting. This percentage varies 


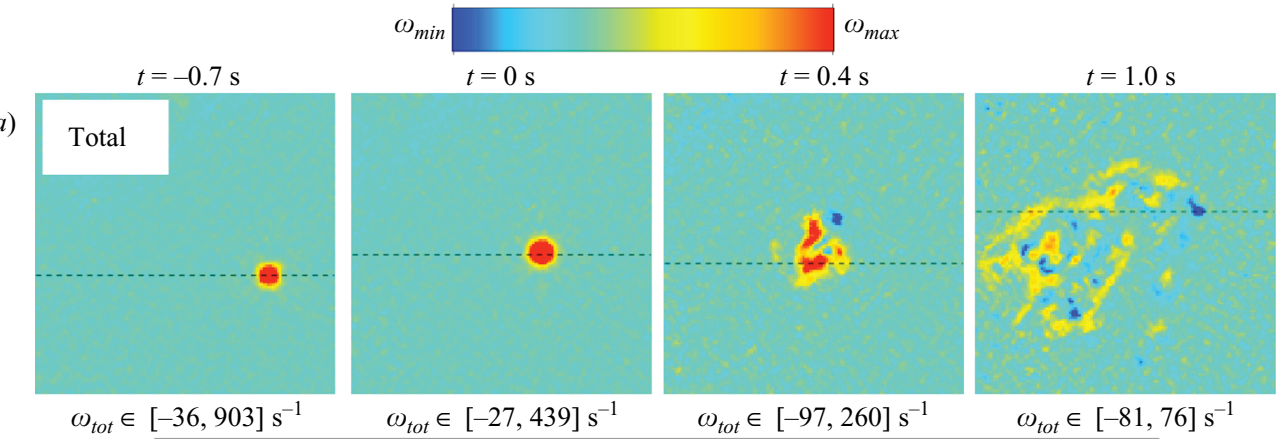

(b)

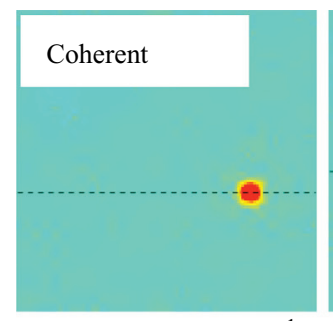

$\omega_{c o h} \in[-26,908] \mathrm{s}^{-1}$
$N_{c o h}=1.3 \%$
$Z_{c o h}=97.0 \%$

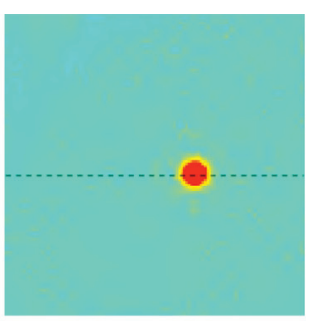

$\omega_{c o h} \in[-21,439] \mathrm{s}^{-1}$

$N_{\text {coh }}=1.4 \%$

$Z_{c o h}=96.3 \%$
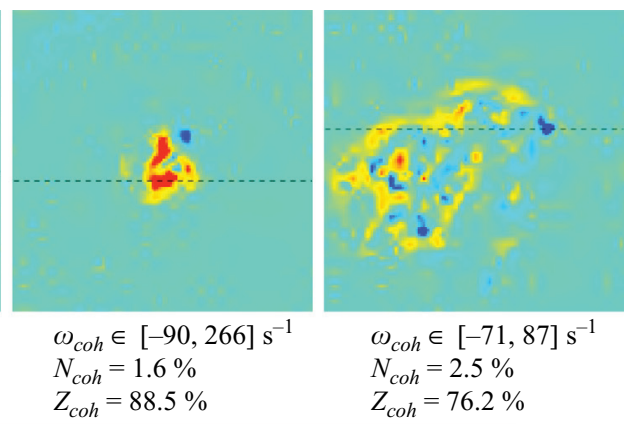

(c)
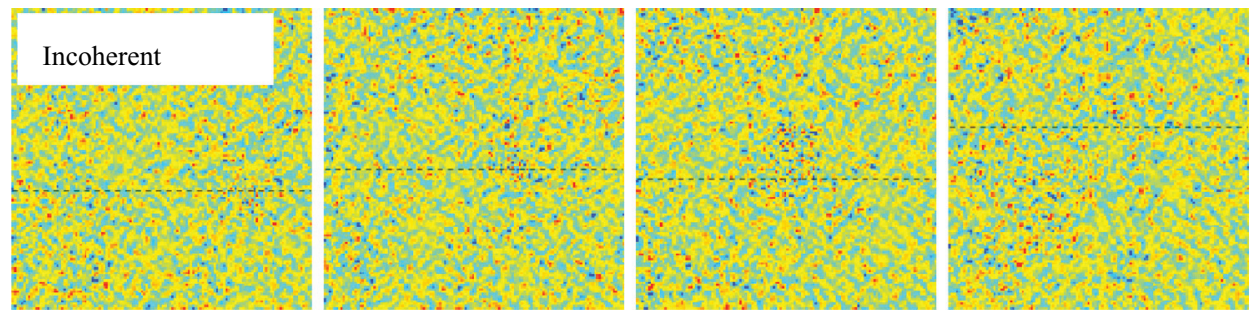

$$
\begin{aligned}
& \omega_{i n c} \in[-25,24] \mathrm{s}^{-1} \\
& N_{\text {inc }}=98.7 \% \\
& Z_{\text {inc }}=3.0 \%
\end{aligned}
$$$$
\omega_{\text {inc }} \in[-19,22] \mathrm{s}^{-1}
$$$$
N_{\text {inc }}=98.6 \%
$$$$
Z_{\text {inc }}=3.7 \%
$$

$$
\begin{aligned}
& \omega_{\text {inc }} \in[-31,27] \mathrm{s}^{-1} \\
& N_{\text {inc }}=98.4 \% \\
& Z_{\text {inc }}=11.5 \%
\end{aligned}
$$

$\omega_{\text {inc }} \in[-29,29] \mathrm{s}^{-1}$

$N_{\text {inc }}=97.5 \%$

$Z_{\text {inc }}=23.8 \%$

(d)

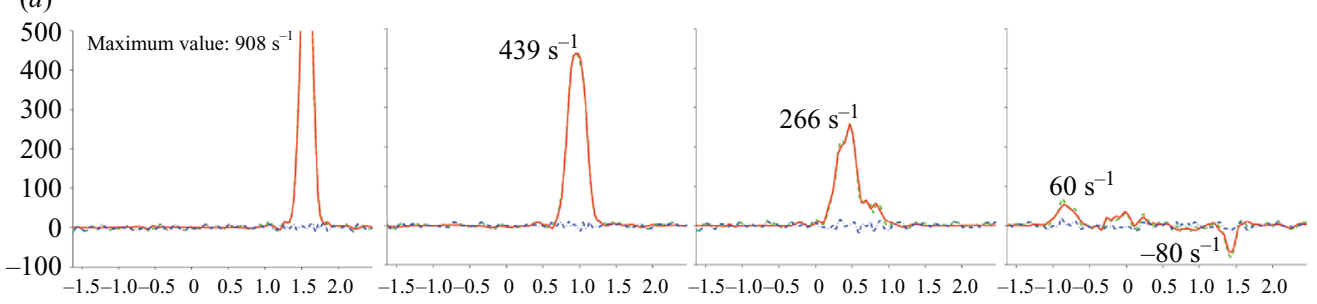

FIGURE 3. Time evolution of the $(a)$ total, $(b)$ coherent and $(c)$ incoherent vorticities at instants $t=\{-0.7,0,0.4,1.0\} \mathrm{s}$, with $t=0$ the beginning of the bursting. The number of coefficients and percentage of enstrophy retained in each component and the extrema of vorticity are indicated below the snapshots. $(d)$ One-dimensional cuts along the vorticity at the locations of the maxima. The dashed green, solid red and dotted-dashed blue lines represent the total, coherent and incoherent fields respectively. (See also movie $3 a$ showing the time evolution of the total vorticity field, movie $3 b$ showing the time evolution of coherent vorticity field and movie $3 c$ showing the time evolution of incoherent vorticity field.) 

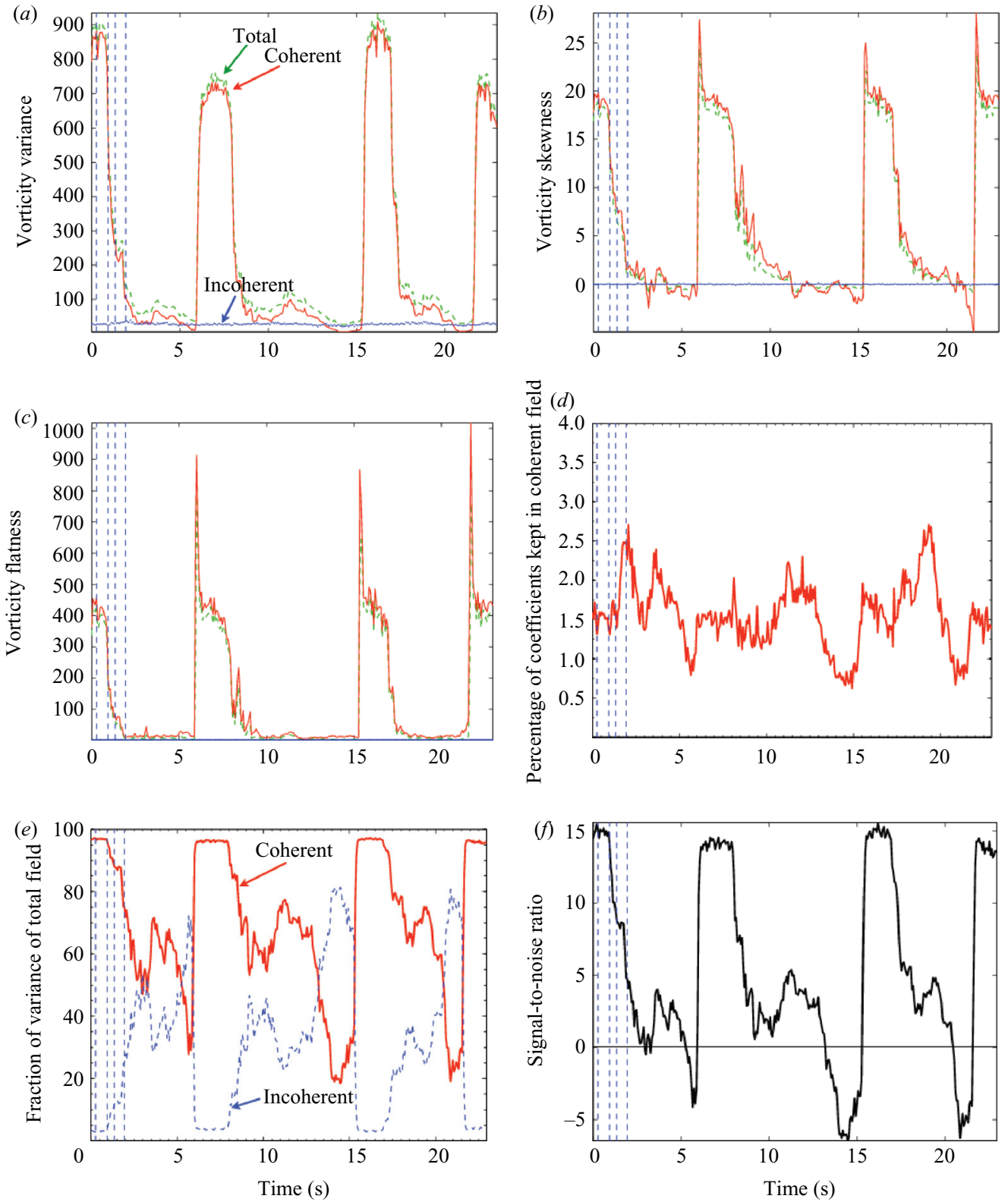

FIgURE 4. Time evolution of different statistical diagnostics for the total (dashed green line), coherent (solid red line) and incoherent (dotted-dashed blue line) vorticities during $23 \mathrm{~s}$, in order to capture three successive bursting events. (a) Variance, (b) flatness, $(c)$ skewness, (d) percentage of orthogonal wavelet coefficients retained in the coherent component, $(e)$ percentage variance of the coherent and incoherent components (sum to $100 \%),(f)$ the coherency measure (CM). (See also movie $4 a$ showing the time evolution of the total, coherent and incoherent vorticities, of the corresponding enstrophy, skewness and flatness, and movie $4 b$ showing the time evolution of the percentage of the number of coherent coefficients, of the percentage of coherent and incoherent enstrophies and of the CM.) 
from $1.3 \% N$, when the vortex is stable, to $2.7 \% N$ just after bursting and falls to $0.7 \% \mathrm{~N}$, when there is no longer any remnant of the vortex in the field of view. Figure 4(e) gives the variation of the percentage of coherent enstrophy, which is a good measure of the dynamical evolution: before the bursting it remains constant around $98 \% Z$ ( $Z$ being the total enstrophy as defined in (3.9)), while just after the bursting it falls down to about $60 \% Z$, and finally below $4 \% Z$ when there is only an incoherent background flow left. The percentage of coherent enstrophy remains approximately constant before the bursting, despite the existence of large variations in the intensity of the successive vortices, as shown in figure 4(e). Likewise, the relative percentage of incoherent enstrophy varies greatly throughout the bursting and is also a good indicator of the dynamical evolution. Indeed, before the vortex bursts it remains negligible, after which it rises to about $40 \% Z$, and later, when there is no longer any vortex or remnant of vortex in view, the incoherent enstrophy contributes a larger percentage to the total enstrophy, up to $70 \% Z$, than the coherent enstrophy.

\subsection{Coherency measure ( $C M$ )}

We define the $\mathrm{CM}$ as the signal-to-noise ratio (3.12) between the coherent and incoherent enstrophies. Its time evolution, shown in figure $4(f)$, exhibits a behaviour similar to the variation in the percentage of enstrophy.

Following several bursts, we observe a typical evolution of $\mathrm{CM}$ which allows us to decompose the vortex bursting into three stages:

(i) during the first stage the vortex remains stable and CM has a constant value about 15 ;

(ii) as soon as the vortex destabilizes, it abruptly drops to values below two, which corresponds to the second stage when vortex bursts and incoherent enstrophy is produced;

(iii) later we observe a progressive increase of $\mathrm{CM}$ up to five which measures the roll-up of vorticity filaments or merging of same-sign vortex structures, characteristic of the third stage.

Finally, the CM becomes negative when the remnants of the vortex bursting are swept out of the observation window and and remains so until the next vortex enters. Thus the $\mathrm{CM}$ gives a precise and quantitative way to characterize and analyse the vortex bursting.

\subsection{Cross-PDF between vorticity and streamfunction}

The spatial coherence of the flow can be revealed by the scatter plot of vorticity versus streamfunction that estimates the cross-probability distribution function (crossPDF) between these fields, as shown in figure 5(a) (see also movie $5 a$ showing its time evolution). For a flow that contains coherent structures, such as vortices, the distribution is organized along branches, each approximating a sinh function, as predicted by Joyce \& Montgomery (1973). The long arm observed in the scatter plot of the total and coherent flows prior to the vortex bursting is similar to a distribution observed for a single Burger's vortex. This is another a posteriori proof that the wavelet method has well extracted the coherent vortex out of the flow. As the bursting proceeds, vorticity extrema are reduced by a factor 100, and the scatter plot distribution contracts near the origin. The scatter plot of the coherent flow exactly follows the evolution of the scatter plot of the total flow. In contrast, the scatter plot of the incoherent flow remains stationary and localized around the origin throughout the bursting, with a maximum value of 25 . After the bursting there remains for a while some coherence due to vortex remnants until the next vortex enters the observation window. 


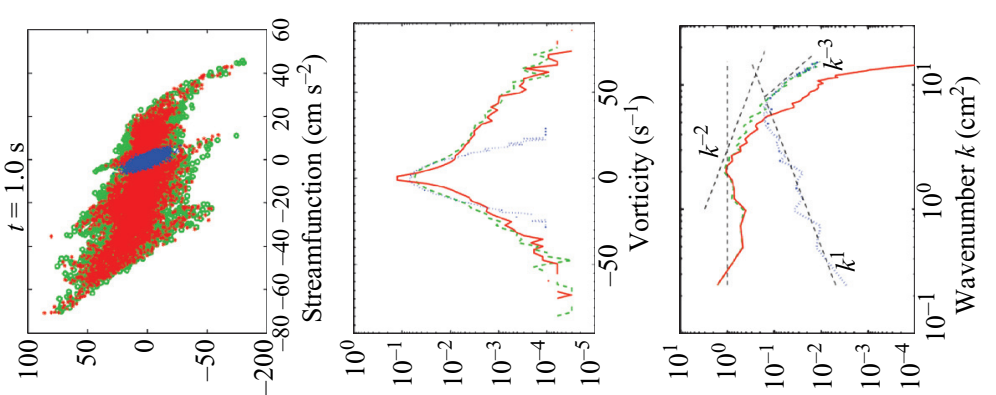

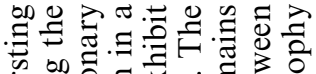

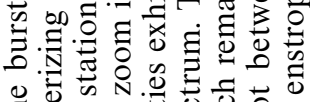

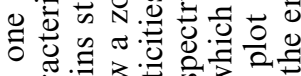

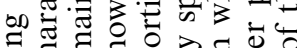

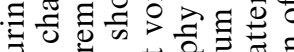

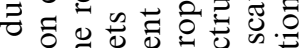

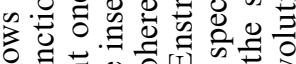

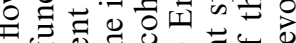
(2)

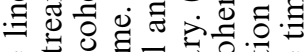

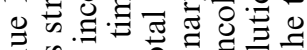

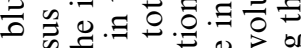
उ己

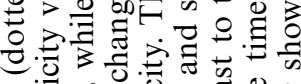

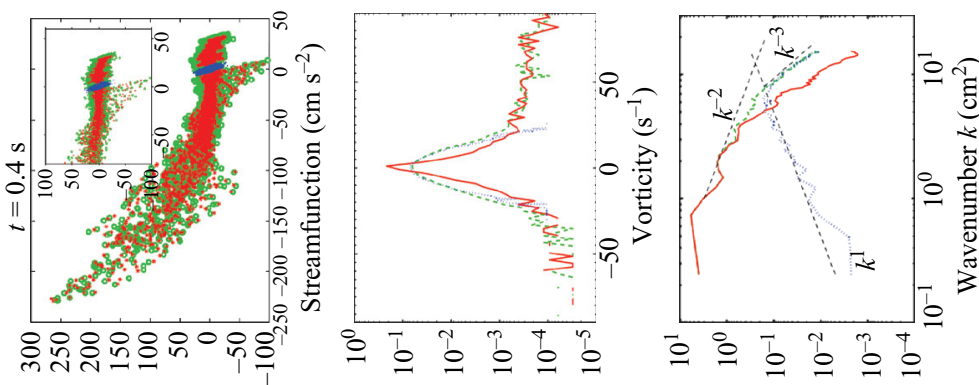

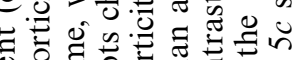
ขั0

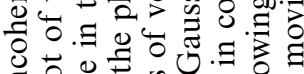

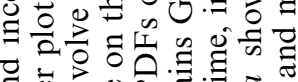

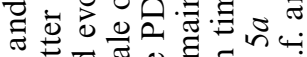

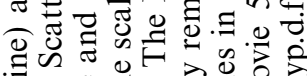

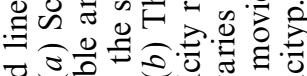
든?

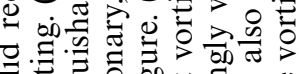

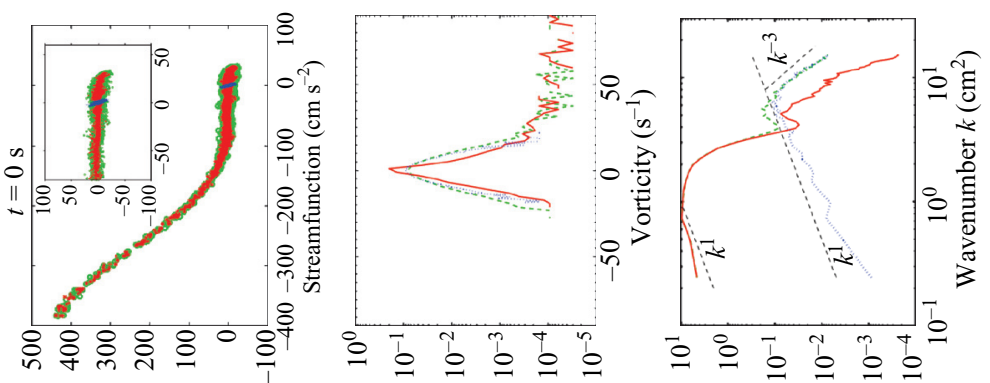

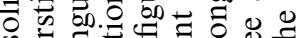

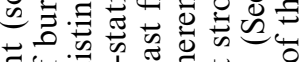

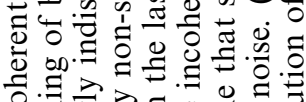

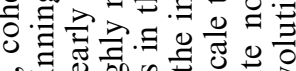

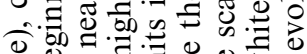

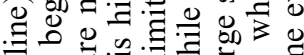

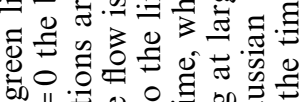

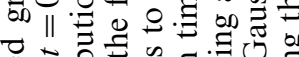

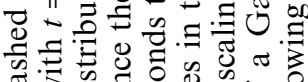

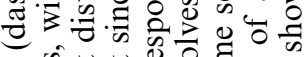

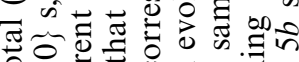

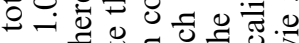

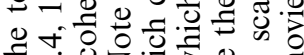

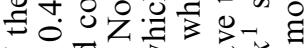

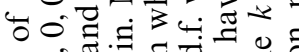

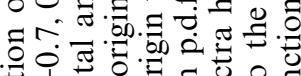

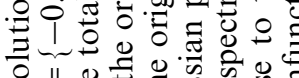

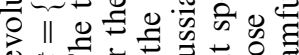

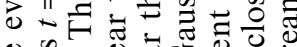

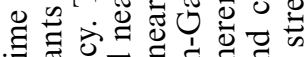

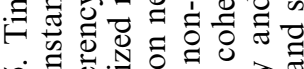

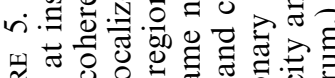

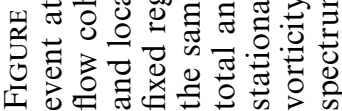




\subsection{Vorticity $P D F$}

Until here we have studied the time evolution of three successive bursting events. We now focus on a single burst and analyse four characteristic instants, as previously defined in $\S 4.3$. We first consider the time evolution of the PDF of vorticity, shown in figure $5(b)$ (see also movie $5 b$ ). The PDF of the coherent vorticity follows closely that of the total vorticity, which is far from Gaussian. Before the bursting the vorticity PDFs are highly skewed, with a stretched positive tail as shown in figures 5(a) and $5(b)$ that corresponds to the isolated vortex of positive sign. After the bursting we observe that the stretched exponential tail is intensified for the positive values, while a weaker tail also develops for negative values, indicating a production of enstrophy during the bursting. By time $t_{4}$ at the end of the bursting, the PDFs of both the total and coherent vorticities have become more symmetric and less narrow than before the bursting. In contrast, the PDF of the incoherent vorticity has remained symmetric and close to Gaussian throughout the bursting process, as seen in figure 5(b) (see also movie $5 b$ ).

\subsection{Enstrophy spectrum}

The time evolution of the enstrophy spectrum is presented in figure 5(c). At early time $t_{1}=-0.7 \mathrm{~s}$, before the vortex bursts, we observe a $k^{+1}$ scaling at large scales and an exponential decay at intermediate scales that corresponds to the Gaussian profile of the isolated vortex, as shown in figure $3(d)$. At time $t_{2}=0 \mathrm{~s}$, we observe that intermediate scales are depleted of their enstrophy to the benefit of smaller scales. At time $t_{3}=0.4 \mathrm{~s}$, the enstrophy transfers from large to intermediate scales filling the gap at intermediate scales, and the enstrophy spectrum tends towards a $k^{-2}$ scaling. At time $t_{4}=1 \mathrm{~s}$, the transfer of enstrophy towards smaller scales goes on and results in a flat spectrum at large scales and a $k^{-2}$ scaling at intermediate scales. All along this evolution, the small scales exhibit a $k^{-3}$ scaling which has probably to do with a noise resulting from the PIV measurements and algorithm.

The spectrum of the coherent enstrophy matches that of the total enstrophy and follows its time evolution at large and intermediate scales. In contrast the incoherent enstrophy spectrum remains stationary and exhibits a $k^{+1}$ scaling, which corresponds to an enstrophy equipartition (since to compute the spectrum we have integrated the two-dimensional spectrum over directions). The incoherent enstrophy remains very low and takes over the coherent enstrophy only at small scales, where we observe a $k^{-3}$ scaling, very probably due to the PIV noise.

We have found that at low wavenumbers the enstrophy spectrum strongly varies in time, exhibiting a scaling between $k^{+1}$ (at the beginning) and $k^{0}$ (at the end of the vortex bursting). If we assume the relation $Z(k)=k^{2} E(k)$ (which cannot strictly hold here, since the measurements are made only in a two-dimensional plane), these results seem to confirm the observations we have previously made for the vortex bursting, where we showed that the slope of the energy spectrum varies in time from $k^{-1}$ to $k^{-2}$ and that the $k^{-5 / 3}$ scaling predicted by Kolmogorov is only obtained for time averages (Cuypers et al. 2003, 2004, 2006).

\section{Results: wavelet analysis and intermittency measures}

\subsection{Continuous wavelet analysis}

We present the results of the continuous wavelet analysis, in particular the local intermittency measure. After splitting each turbulent flow realization into coherent and incoherent components, we analyse their contribution independently. 

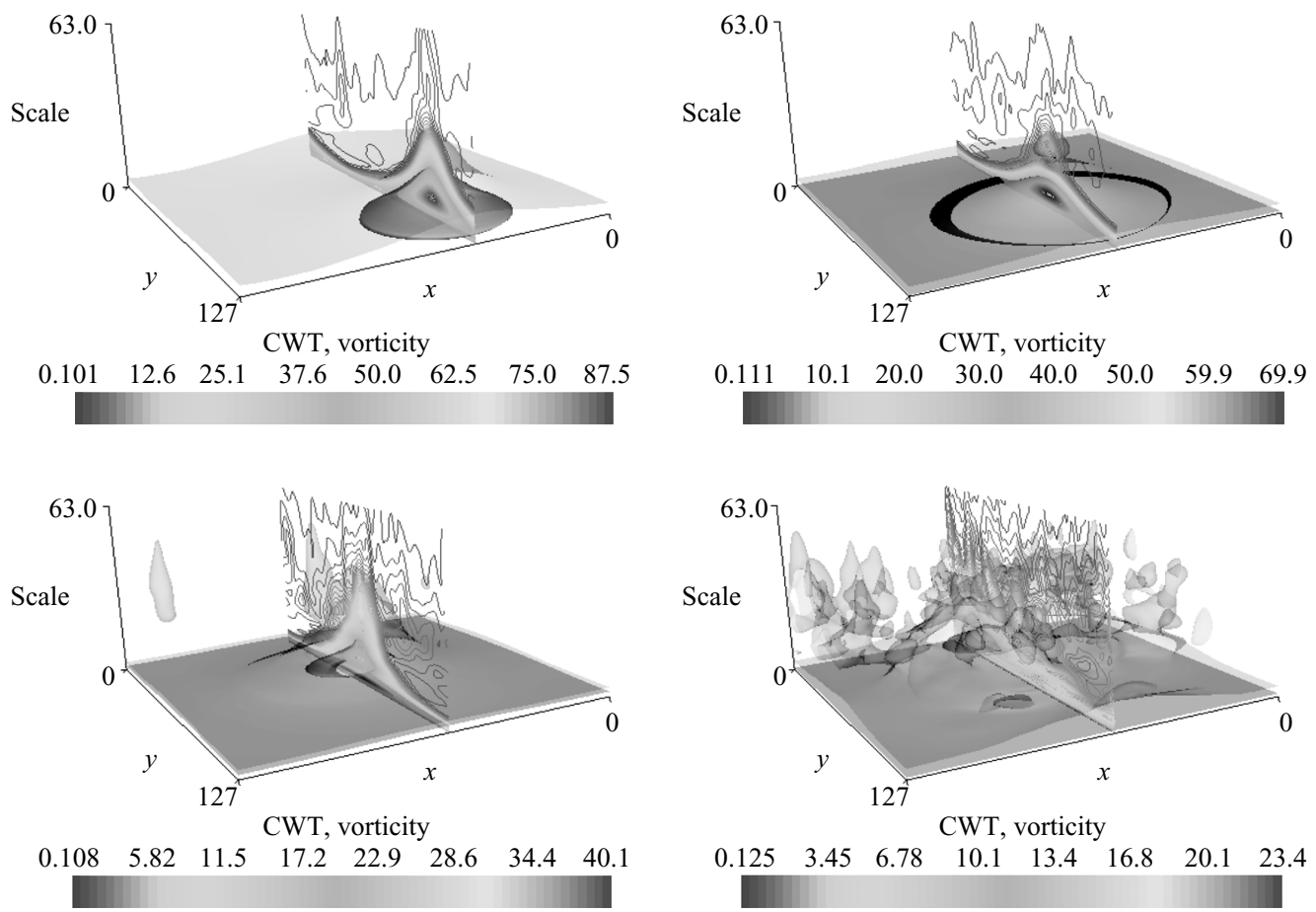

Figure 6. Modulus of the CWT coefficients of the vorticity field at instants $t=$ $\{-0.7,0,0.4,1.0\} \mathrm{s}$, with $t=0$ the beginning of the bursting. The isosurfaces correspond to $50 \%$ and $10 \%$ of the maximum values indicated on the colour scales. The horizontal axes give the position and the vertical axes the scale, with the smallest scales at the top. The plane cut slices the position-scale plane at the maximum value of the CWT coefficients. (See also movie $6 a$ showing the time evolution of the modulus of the CWT coefficients in the whole wavelet space and movie $6 a$ showing the time evolution of the modulus of the CWT coefficients in a two-dimensional cut which tracks the vortex.)

We calculate the CWT of the coherent vorticity field, shown in figure 6 (and in movies $6 a$ and $6 b$ ), using a complex-valued Morlet wavelet. Due to Parseval's relation (2.7), the square modulus of the wavelet coefficients gives the local enstrophy density in both space and scale, from which we compute the LIM, i.e. the spatial variability of the enstrophy at each scale or likewise the spatial deviation from the mean enstrophy spectrum (Farge 1992). We can thus identify which space-scale regions actively contribute to the nonlinear cascade and which space-scale regions are dominated by viscous dissipation, as seen in figure 7 (and in movies $7 a$ and $7 b$ ).

As a complement to the PIV measurements, we also study the time evolution of a dye injected into the flow to analyse the vortex bursting using a much faster camera which captures 1000 frames per second instead of 15 double frames per second. (Movie $8 a$ shows $6 \mathrm{~s}$ of the flow evolution which has been slowed down to the last $13 \mathrm{~s}$ in order to better understand the bursting process.)

\subsection{Modulus of the continuous wavelet coefficients}

The modulus of the CWT coefficients of vorticity are shown in figure 6 for the four time snapshots defined in $\S 4.3$ (see also movies $6 a$ and $6 b$ ). Before the bursting, the largest values of the CWT coefficients are located at the vortex core (see $t_{1}$ in 

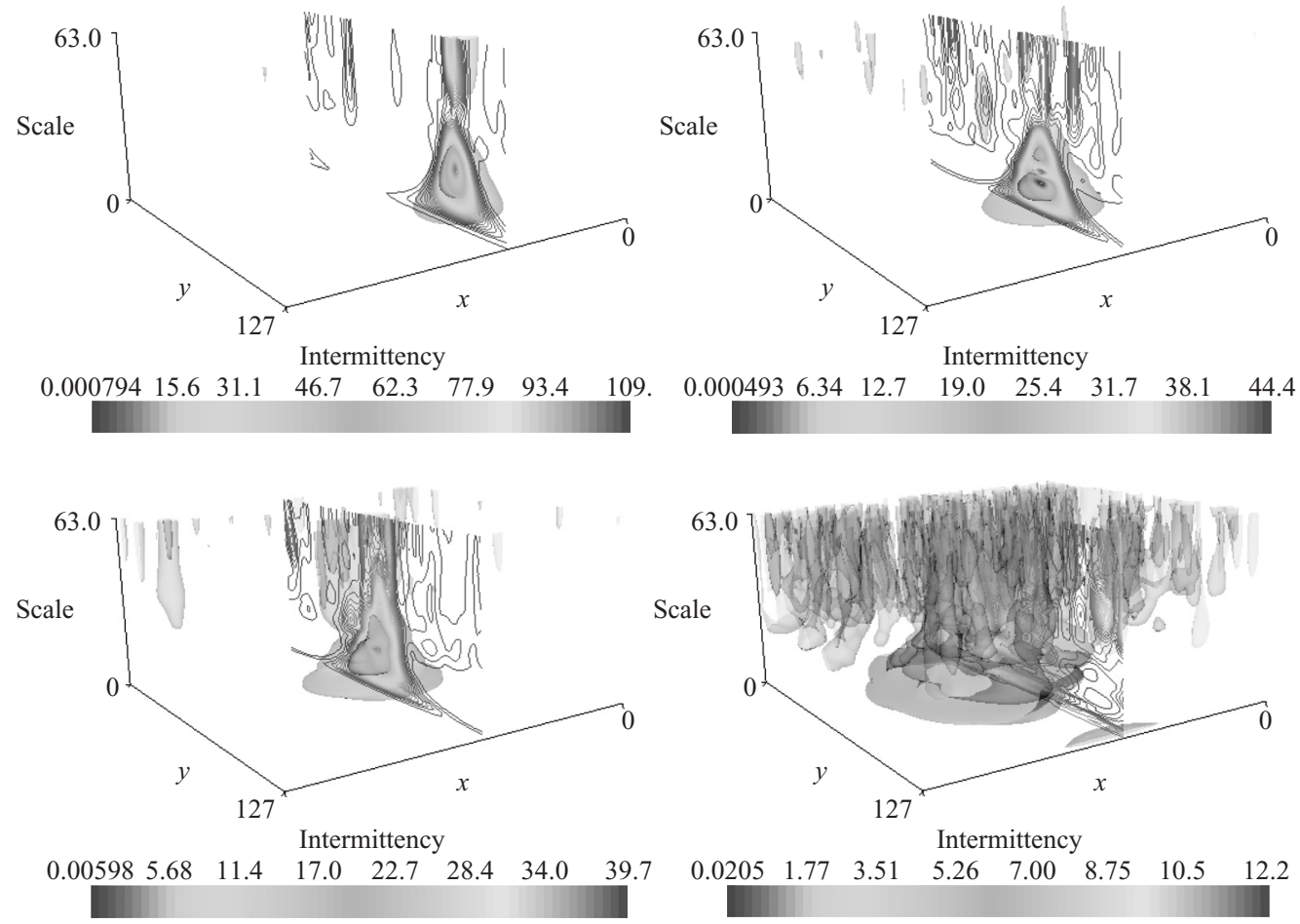

FIGURE 7. The LIM of the vorticity field at instants $t=\{-0.7,0,0.4,1.0\} \mathrm{s}$, with $t=0$ the beginning of the bursting. The isosurfaces correspond to $50 \%$ and $10 \%$ of the maximum values indicated on the colour scales. The horizontal axes give the position and the vertical axis the scale, with the smallest scales at the top. The plane cut slices the position-scale plane at the maximum value of the LIM. (See also movie $7 a$ showing the time evolution of the LIM in the whole wavelet space and movie $7 b$ showing the time evolution of the LIM after averaging the LIM in scale.)

figure 6). This confirms that the large values in the enstrophy spectrum at large scales are due to the spatially localized contribution which corresponds to the support of the vortex. At time $t_{2}$, the very beginning of the bursting, the modulus of the CWT coefficients starts decreasing and its maximal values, around 70, shift towards smaller wavenumbers, which indicates an enstrophy transfer towards smaller scales. We simultaneously observe the vortex spatially expanding while keeping its shape. By time $t_{3}$, as the enstrophy cascade is developing, the vortex core begins to break up into several pieces, which is denoted by the emergence of several local maxima of the modulus of the CWT coefficients. Finally, by the end of the bursting, the values of the large-scale wavelet coefficients continue to decrease, since the enstrophy is transferred towards smaller scales (figure $5 c$ ), while their support spreads out in both space and scale (figure 6). By time $t_{4}$ the magnitude of the wavelets coefficients is now below 20 , and a significant portion of enstrophy has reached the small scales.

The continuous wavelet analysis of the vorticity field, measured by PIV using a standard camera, has revealed that the bursting process starts in the vortex core. The time evolution of a dye advected by the vortex and recorded (in a two-dimensional plane perpendicular to the vortex axis) with a camera, 66 times faster than the camera used for PIV, confirms that the destabilization of the vortex starts from the core. In movie 8(a) we observe that the dye is advected by the vortex and exhibits a stable 
structure spiralling around its centre. Later the vortex centre begins to describe a circular motion in the clockwise direction, and very locally the dye starts to unwrap but without affecting the rest of the dye. Soon after, a dislocation appears in the spiral pattern of the dye, and several vortical structures emerge that unwrap the dye in the counterclockwise direction, which then mixes it all over the place. We have also performed a continuous wavelet analysis of the dye concentration field as recorded at 1000 frames per second (see movies $8 a$ and $8 b$ ), which confirms that the bursting process starts from the vortex core.

Since we observe the flow only in a plane perpendicular to the vortex axis, it is impossible at this stage to obtain a more complete description of the bursting process using PIV. We tried to perform PIV measurements in a horizontal plane parallel to the vortex axis, but we did not succeeded, since the vortex does not remain in this plane and the velocity gradients are much too large to guarantee enough precision of the velocity measurement in the horizontal plane. By observing the time evolution of dyes, having four different colours, injected into the vortex at four locations and recorded from above in a horizontal plane parallel to the vortex axis (see movie 1) using a standard camera, we get the feeling that the vortex is made of several vortex tubes nested within each others which alternate opposite-sign helicities. The bursting seems to happen when the connection with at least one suction hole is lost. Since the PIV measurements are performed in the central plane, as shown in figure $1(a)$, far away from the suction holes, it is very difficult to propose a precise scenario, i.e. to decide if the bursting is due to the bending of the vortex tube while it is pushed downstream by the laminar flow, due to the stretching imposed by the suction at the sidewalls or due to the competition between both mechanisms.

\subsection{Local intermittency measure}

The local intermittency measure (LIM) of vorticity corresponds to the modulus of the CWT coefficients of vorticity renormalized at each scale by the enstrophy content at that scale. The LIM is thus more appropriate than the wavelet coefficients to display scale-dependent data over a wide range of scales, since it is simpler to visualize being a relative (renormalized) quantity whose range of variation in intensity is reduced. The LIM also better detects intermittent features because it enhances them more than the wavelet coefficients themselves. In regions in which wavelet coefficients are possibly large but their small-scale contribution is homogenenously spread in space, there is no intermittency and the LIM remains close to one everywhere. In regions in which the spatial distribution of the wavelet coefficients becomes sparser towards small scales, the LIM reaches high values, which quantify how much the variance locally exceeds the mean variance at a given scale. The LIM thus gives a quantitative estimation of intermittency.

The LIM of vorticity is shown in figure 7 . We observe that at early time, before the vortex bursting, the LIM presents large values in a wide range of scales that are concentrated in a small region around the vortex core. This confirms that an isolated stable vortex is an intermittent structure, which is smooth, since the maximum of the LIM, which is above 100, remains at large scales. When the vortex bursts, the maximum LIM value decreases to about 40, and we observe a spreading at small scales indicating the turbulent cascade and the concomitant production of more singular features. From there on the maximum value of the LIM continues to decrease, reaching a maximum of 30 by $t_{3}=0.4 \mathrm{~s}$ and 10 around $t_{4}=1.0 \mathrm{~s}$, while the small-scale activity is spreading out in space, e.g. we observe that the LIM isosurface $I=2$ then fills most of the space at small scales. 
Before bursting

(a)

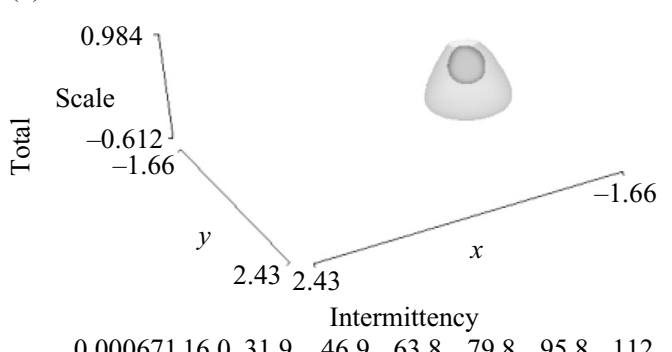

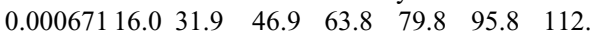

(b)

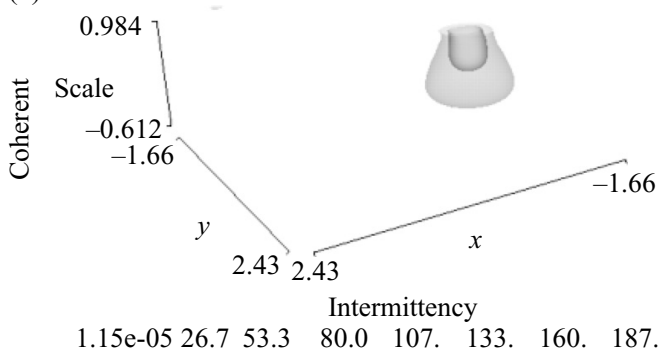

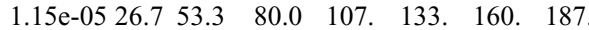

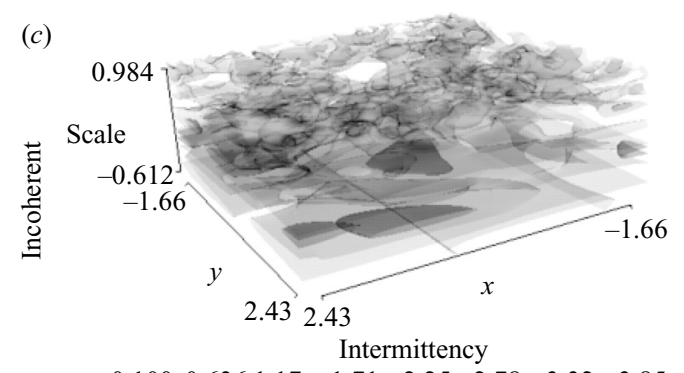

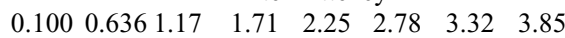

After bursting

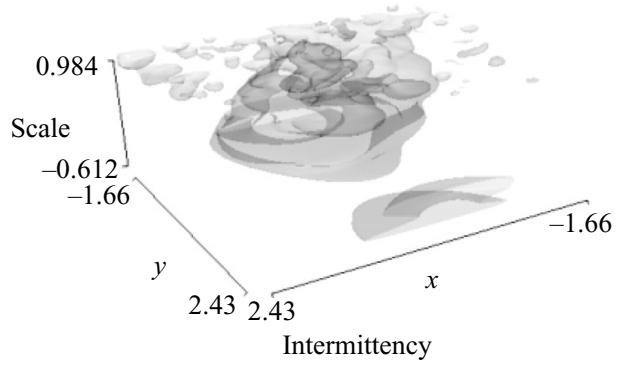

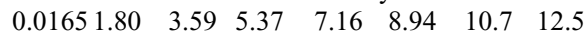

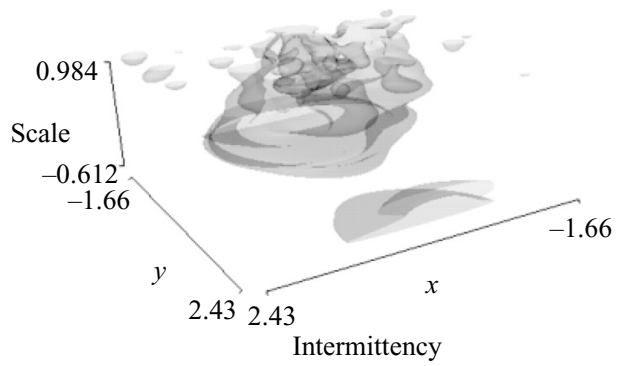

$\begin{array}{llllllll}2.55 \mathrm{e}-06 & 2.85 & 5.70 & 8.55 & 11.4 & 14.2 & 17.1 & 19.9\end{array}$

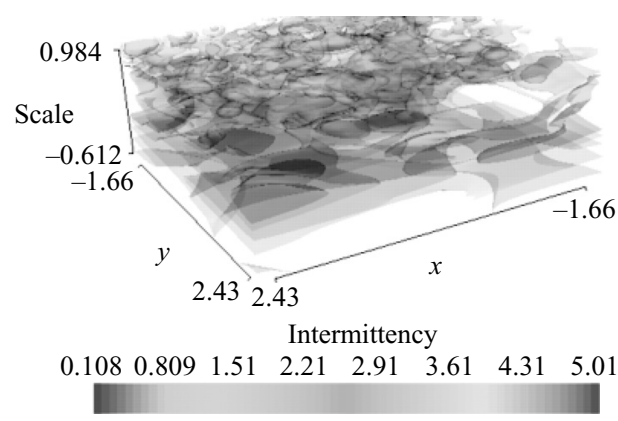

FIGURE 8 . The LIM before (left) and after (right) the bursting for the $(a)$ total, $(b)$ coherent and $(c)$ incoherent vorticities. The isosurfaces correspond to $50 \%$ and $10 \%$ of the maximum values indicated on the colour scales. The horizontal axes give the position and the vertical axis the scale, with the smallest scales at the top.

In figure 8 we show the evolution of the LIM before and after the bursting for the total, coherent and incoherent vorticities. When the vortex is isolated and stable, the LIM is maximal in the vortex core, where it reaches values up to 187 for the coherent flow (figure $8 b$, left) but only up to 112 for the total flow (figure $8 a$, left) whose intermittency is reduced due to the presence of experimental PIV noise (figure $8 c$, left). When the vortex bursts, the LIM suddenly falls down, with its maximal value reduced to 20 for the coherent flow (figure $8 b$, right) and to 12 for the total flow (figure $8 a$, right), which is weaker than for the coherent flow, since the total flow 
also contains the incoherent flow produced by the vortex bursting, together with experimental PIV noise (figure $8 c$, right).

\section{Conclusion}

We have proposed several diagnostics to statistically analyse highly unsteady and inhomogeneous turbulent flows. To illustrate the potential of these wavelet methods, we have chosen to study the bursting of a three-dimensional vortex, advected by a laminar flow and subjected to stretching, measured by PIV.

We have first applied a wavelet-based eduction method to extract coherent structures out of turbulent flows. We have thus found that very few orthogonal wavelet coefficients are sufficient to track the vortex bursting and its nonlinear evolution, preserving in particular the vorticity PDF, the enstrophy spectrum in the inertial range and the cross-PDF between vorticity and streamfunction (which is a good diagnostic for coherence). The number of strong wavelet coefficients, which correspond to the coherent structures, varies during the flow evolution between $1.3 \%$ and $2.7 \%$ of $N$ ( $N$ being the resolution of the PIV image). The remaining weakest wavelet coefficients, whose number varies between $97.3 \%$ and $98.7 \%$ of $N$, take care of the experimental noise and of the incoherent background flow. This method is simple, since there is no parameter to adjust besides the choice of the orthogonal wavelet basis, and more universal than other eduction methods, since it does not depend on the flow configuration. Note that if the flow is not turbulent enough, all modes are coherent. We have proposed in this paper the use of the incoherent enstrophy as a good estimate of the turbulence level.

We have also proposed a new diagnostic, the coherency measure $(\mathrm{CM})$, which is defined as the signal-to-noise ratio between the coherent and incoherent enstrophies. It is used to quantitatively characterize the different stages of the vortex bursting. Following its evolution, we have identified three characteristic ranges of values that the CM takes before, during and after the vortex bursting. At first, when the vortex evolves as a stable isolated structure, the CM has a large positive value. Then it abruptly drops to small positive values at the instant of bursting, and later it slightly increases when the vorticity filaments and the vorticity sheets produced by the bursting begin to reorganize themselves by nonlinear interaction. Finally, the CM becomes negative when the remnants of the vortex bursting are swept out of the field of view, and it remains so until a new vortex enters the observation window. The vortex life cycle then reproduces itself.

To complement the CM, we have designed other diagnostics based on the continuous wavelet transform. Our aim is to better understand the build-up of the turbulent cascade and to find with them when and where, in both space and scale, the nonlinear activity is dominant. Using the local intermittency measure (LIM), we found that the incoherent flow is non-intermittent (the LIM always remains below 5), exhibits an enstrophy equipartition spectrum and has a quasi-Gaussian p.d.f. of vorticity. In contrast, the coherent flow is intermittent (the LIM reachs values up to 160) and sustains the turbulent cascade, with a correlated enstrophy spectrum in the inertial range and a non-Gaussian vorticity PDF.

The continuous wavelet coefficients show that the bursting starts as an excitation of the small scales of the vortex core and then spreads in space and scale. The wavelet representation is optimal to reveal such a localized scaling phenomenon, and the LIM is the appropriate diagnostic to study it. This result has been confirmed by dye visualization recorded with a very fast camera to decompose 
the motion just before the instant of bursting. We have also observed that before the vortex bursts, its coherent component retains $98 \%$ of the total enstrophy $Z$, a proportion which suddenly drops to about $60 \%$ of $Z$ when the vortex bursts and later becomes negligible as the remnants of the vortex are swept out of the observation window.

We found that the coherent enstrophy spectrum is highly non-stationary and follows the total enstrophy evolution. In contrast the incoherent enstrophy remains steady, with a $k^{+1}$ scaling all along the inertial range that corresponds to an enstrophy equipartition. We have thus managed to disentangle a steady, homogeneous and decorrelated background flow from an unsteady, inhomogeneous and correlated flow. The former corresponds to the vortex nonlinear dynamics, while the latter is a good indicator of the turbulence level.

In conclusion, we think that the definition of coherent structures and the waveletbased eduction method we have proposed overcome the problems encountered by previous methods used to extract coherent structures. Using them, we have shown that coherent and incoherent flows are active all along the inertial range, i.e. at both large and small scales, but exhibit different scaling laws, namely long-range correlation for the former and decorrelation for the latter. The results presented in this paper thus confirm the conjecture proposed in Hussain (1986) that 'incoherent turbulence does not consist of only fine-scale turbulence, as is generally presumed, but may contain large-scale irrotational (perhaps even vortical but irrelevant) motions.' We also fully agree with Hussain (1986) when he adds, 'The interaction between coherent structures and incoherent turbulence is the most critical and least understood aspect of turbulent shear flows. This coupling appears to be rather different from the classical notion of cascade; even considering the large and fine scales, they are not decoupled as widely presumed.' This lack of scale separation is the central difficulty encountered in modelling turbulence. The wavelet-based tools presented here offer new ways to handle that problem, and hopefully overcome it.

The authors would like to thank Kai Schneider, Agnès Maurel and Piotr Boronski for useful discussions, together with Alan Norton and John Clyne who provided the vapor visualization package (www.vapor.ucar.edu) that has been used for the threedimensional visualizations. JRF thanks the Ministère de la Recherche and the Agence Nationale pour la Recherche (ANR) for financing his post-doctoral research. MF is grateful to the Newton Institute for Mathematical Sciences (Cambridge, UK), where she completed the revised version of this paper, and to the Association CEA-Euratom for supporting her research under contract no. V.3258.001.

\section{REFERENCES}

Antonia, R. A. 1981 Conditional sampling in turbulence measurement. Annu. Rev. Fluid Mech. 13, $131-156$.

Azzalini, A., Farge, M. \& Schneider, K. 2005 Nonlinear wavelet thresholding: a recursive method to determine the optimal denoising threshold. Appl. Comput. Harmon. Anal. 18, 177-185.

Berkooz, G., Holmes, P. \& Lumley, J. L. 1993 The proper orthogonal decomposition in the analysis of turbulent flows. Annu. Rev. Fluid Mech. 25, 539-575.

Blackwelder, R. F. 1977 On the role of phase information in conditional sampling. Phys. Fluids 20, S232.

Cantwell, B. J. 1981 Organized motion in turbulent flow. Annu. Rev. Fluid Mech. 13, 457-515.

Cuypers, Y., Maurel, S. \& Petitjeans, P. 2003 Vortex burst as a source of turbulence. Phys. Rev. Lett. 91 (19), 194502. 
Cuypers, Y., Maurel, A. \& Petitjeans, P. 2004 Comparison between an experimental turbulent vortex and the Lundgren vortex. J. Turbulence 5, 30.

Cuypers, Y., Maurel, A. \& Petitjeans, P. 2006 Characterization of an experimental turbulent vortex in the physical and spectral spaces. J. Turbulence 7, 7.

Donoho, D. \& Johnstone, I. 1994 Ideal spatial adaptation via wavelet shrinkage. Biometrika 81, 425-455.

FARGE, M. 1992 Wavelet transforms and their applications to turbulence. Annu. Rev. Fluid Mech. 24, 395-457.

Farge, M., Goirand, E., Meyer, Y., Pascal, F. \& Wickerhauser, M. V. 1992 Improved predictability of two-dimensional turbulent flows using wavelet packet compression. Fluid Dyn. Res. 10, 229-250.

Farge, M., Guezennec, Y. Ho, C. M. \& Meneveau, C. 1990 Continuous wavelet analysis of coherent structures. In Stanford University, Studying Turbulence Using Numerical Simulation Databases. 3: Proceedings of the 1990 Summer Program (pp. 331-348).

FARGE, M. \& RABREAU, G. 1988 Transformée en ondelettes pour détecter et analyser les structures cohérentes dans les écoulements turbulents bidimensionnels. C. R. Acad. Sci. Paris 307 (série II), 1479-1486.

Farge, M., Pellegrino, G. \& Schneider, K. 2001 Coherent Vortex Extraction in three-dimensional Turbulent Flows Using Orthogonal Wavelets. Phys. Rev. Lett. 87 (5), 054501.

Farge, M., Pellegrino, G. \& Schneider, K. 2003 Coherent Vortex Extraction in threedimensional homogeneous turbulence: comparison between CVS-filtered and POF-Fourier decompositions. Phys. Fluids 15 (10), 2886-2896.

Farge, M., Schneider, K. \& Kevlahan, N. 1999 Non-Gaussianity and coherent vortex simulation for two-dimensional turbulence using an adaptive orthogonal wavelet basis. Phys. Fluids 11, $2187-2201$.

Fiedler, H. E. 1988 Coherent structures in turbulent flows. Prog. Aerosp. Sci. 25, 231-269.

Hussain, A. K. M. F. 1986 Coherent structures and turbulence. J. Fluid Mech. 173, 303-356.

JoyCe, G. \& Montgomery, D. 1973 Negative temperature states for the two-dimensional guidingcentre plasma. J. Plasma Phys. 10, 107.

Kolmogorov, A. N. 1941 The local structure of turbulence in an incompressible fluid at very high Reynolds numbers. Dokl. AN SSSR 30, 299-303.

Lundgren, T. S. 1982 Strained spiral vortex model for turbulent fine structure. Phys. Fluids 25, 2193-2203.

Mallat, S. G. 1989 Multiresolution approximation and wavelet orthogonal bases of $L^{2}(\mathbb{R})$. Trans. Am. Math. Soc. 315.

Meneveau, C. 1991 Analysis of turbulence in the orthonormal wavelet representation. J. Fluid Mech. 232, 469-520.

Robinson, S. K. 1991 Coherent motions in the turbulent boundary layer. Annu. Rev. Fluid Mech. 23, 601-639.

Ruppert-Felsot, J. E., Praud, O., Sharon, E. \& Swinney, H. L. 2005 Extraction of coherent structures in a rotating turbulent flow experiment. Phys. Rev. E 72(1), 016311. 